\title{
Probing the reheating phase through primordial magnetic field and CMB
}

\author{
Md Riajul Haque, ${ }^{*}$ Debaprasad Maity $\odot,^{\dagger}$ and Sourav Pal ${ }^{\ddagger}$ \\ Department of Physics, Indian Institute of Technology Guwahati, Guwahati, Assam, India
}

(Received 30 December 2020; accepted 30 April 2021; published 28 May 2021)

\begin{abstract}
Inflationary magnetogenesis has long been assumed to be the most promising mechanism for producing the large-scale magnetic fields in our universe. However, generically, such models are plagued with either backreaction or strong coupling problems within the standard framework. This paper has shown that the reheating phase can play a crucial role in alleviating those problems along with the cosmic microwave background. The electrical conductivity is assumed to be negligible during the entire period of reheating, and the classic Faraday electromagnetic induction changes the magnetic field's dynamics drastically. Our detailed analysis reveals that this physical phenomenon not only converts a large class of magnetogenesis model observationally viable without any theoretical problem but also can uniquely fix the perturbative average inflaton equation of state, $\omega_{\phi}=(p+2) /(p+2)$ during reheating given a specific value of the large scale magnetic field. This observation hints the inflaton to assume the potential of form $V(\phi) \sim \phi^{p}$ near its minimum with $p \gtrsim 3.5$ if one considers the limitations of the present-day strength of the large scale magnetic field to be $\mathcal{P}_{B 0}^{\frac{1}{2}} \gtrsim 10^{-18}$ G. Our analysis opens up a new avenue toward constraining the inflationary and magnetogenesis model together via reheating.
\end{abstract}

DOI: 10.1103/PhysRevD.103.103540

\section{INTRODUCTION}

Reheating is one of the most important early phases of our universe. It essentially links the standard thermal universe with its prethermal phase, namely the inflationary universe, through a complicated nonlinear process. Over the years, major cosmological observations [1-4] have given us ample evidences in understanding the theoretical and observational aspects of both the thermal and the nonthermal inflationary universe cosmology to an unprecedented label. However, the intermediate reheating phase is still to be understood in terms of theory and observation. From the cosmic microwave background (CMB) anisotropy [1], one can estimate the baryon content of the universe, which agrees extremely well with the theoretical prediction of big bang nucleosynthesis (BBN) [5-8]. Furthermore, with the successful standard big bang model, we have a very good understanding over a large timescale of the universe from the present (redshift $z=0$ ) to BBN stage $\left(z \sim 10^{9}\right)$ at an energy scale $\sim \mathcal{O}(1) \mathrm{MeV}$. The tiny fluctuation of CMB anisotropy can be successfully linked

\footnotetext{
*riaju176121018@iitg.ac.in

†debu@iitg.ac.in

"pal.sourav@iitg.ac.in
}

Published by the American Physical Society under the terms of the Creative Commons Attribution 4.0 International license. Further distribution of this work must maintain attribution to the author(s) and the published article's title, journal citation, and DOI. Funded by SCOAP ${ }^{3}$. with the almost scale-invariant density fluctuation predicted by the inflation in the early Universe $[9,10]$. Therefore, precession $\mathrm{CMB}$ data has provided us significant insight into how our universe evolves during inflation. However, our goal here is to understand the intermediate phase, through which the universe must undergo after the end of inflation. This phase is largely ill-understood due to the lack of observational evidence. It is generically described by the coherently oscillating inflaton and its nonlinear decay into the radiation field. In the Boltzmann description, the phase is parametrized by the reheating temperature $\left(T_{r e}\right)$ and the reheating equation of state $\left(\omega_{r e}\right)$. Still now both the parameters remain unconstrained except the reheating temperature which is approximately bounded within $10^{15} \mathrm{GeV}>T_{r e}>T_{B B N} \sim 10 \mathrm{MeV}$. However, if one takes into account nonperturbative reheating in the beginning, the upper bound on reheating temperature could be within $10^{10}-10^{13} \mathrm{GeV}$ [11]. Attempts to understand this phase in the literature can be broadly classified into two categories: (i) Studying the background dynamics during reheating reveals useful information about the deep connection among the inflationary scalar spectral index $\left(n_{s}\right)$ and the reheating temperature $\left(T_{r e}\right)$, and reheating equation of state $\left(\omega_{r e}\right)$ [12-15]. (ii) Evolution of inflationary stochastic gravitational waves has been shown to encode valuable information when passing through this phase $[16,17]$.

In this paper, we consider the present-day large scale magnetic field (LSMF) combined with the CMB anisotropy to probe the reheating phase of the universe followed by the 
standard inflationary phase. For LSMF we consider simple model of primordial magnetogeneis [18-21]. While probing the reheating phase through those observables, we further show how the observables can, in turn, constrain the magnetogenesis model itself. Inflationary magnetogenesis models have been studied quite extensively in the literature [21-28]. Mechanisms known so far is essentially related to the way of breaking the conformal invariance in the electromagnetic sector. This conformal breaking theory generically suffers from either strong coupling, or backreaction problems $[29,30]$ which will be elaborated as we go along. In this regard reheating phase has recently been shown to indirectly play a very important [18] role. As stated earlier, the primary motivation of our present study will be to see in detail how the problems can be resolved partially by the reheating phase for various inflationary models and simultaneously provide constraints on the reheating. Taking into account both the $\mathrm{CMB}$ anisotropic constraints on the inflationary power spectrum and the present value of the large scale magnetic field, our analysis reveals an important connection among the reheating parameters $\left(T_{r e}, w_{r e}\right)$, magnetogensis models and inflationary scalar spectral index $\left(n_{s}\right)$.

The universe is observationally shown to be magnetized over a wide range of scales. Zeeman splitting, synchrotron emission, and Faraday rotation are some of the fundamental physical mechanisms by which the existence of a magnetic field can be probed. Various astrophysical and cosmological observations of those quantities tell us that our universe is magnetized over scales starting from our earth, the sun, stars, galaxies, galaxy clusters, and the intergalactic medium (IGM) in voids. In the galaxies and galaxy clusters of few to hundred-kilo parsecs $(\mathrm{kpc})$ scale, the magnetic fields have been observed to be of order a few micro Gauss [31-33]. The $\gamma$-ray observations of GRB 190114C, the observed long-term GeV-TeV light curve of the BLAZAR MRK 421 and BLAZAR MRK 501 suggest that even the intergalactic medium (IGM) in voids can host a weak $\sim 10^{-16}-10^{-20}$ Gauss magnetic field, with the coherence length as large as Mpc scales [34-43]. Furthermore, future space-based $\gamma$-ray observatories such as MAST with improved sensitivity will be able to probe the strength of the extra-galactic magnetic field (EGMF) even below $B<$ $10^{-18} \mathrm{G}$ using the pair echo method [44]. Additionally, Jedamzik et al. [45] derive an upper limit on the PMFs, which is $\sim 47 \mathrm{pG}$ for scale-invariant PMFs, using MHD computation. Therefore, all these efforts and observations hifnt at the importance of understanding large-scale magnetic fields in the theoretical framework. In this context, our main focus of the paper would be to understand the evolution of large-scale magnetic fields. We will not concentrate on the magnetogenesis scenario, which has already been studied quite extensively in the literature [21-24,26]. As the mechanism of inflationary magnetogensis is essentially the same, we will specifically consider the well-known Ratra model [20] for our study. Once the electromagnetic field is generated during inflation, the subsequent evolution occurs through the time-evolving plasma. The magnetic field at the cosmological scale can evolve and survive even today while passing through the plasma state[46,47] of our universe before the structure formation. Therefore, the time-evolving plasma proves to be an ideal environment to have a sustainable evolution and growth of the magnetic field. Usual magnetic hydrodynamic evolution in the time-evolving plasma requires a tiny seed initial magnetic field. In the cosmological context, the well-known mechanism for such seed field is primordial inflationary magnetogenesis. Inflation provides us a unique mechanism for producing coherent magnetic fields for a wide range of scales. Mpc scale magnetic field can survive until today as a cosmological relic whose magnitude could be $\sim 10^{-9}-10^{-20} \mathrm{G}$. On the other hand, at small scales, this tiny inflationary magnetic field can play as a seed field which will be further enhanced by the well-known Galactic dynamo mechanism [32,48-51]. A similar mechanism can also be obtained from the electroweak phase transition (EWPT) [52-55].

We consider a standard scenario of inflationary magnetogenesis where the kinetic term of the electromagnetic field is conformally coupled with a scalar field. Background inflation dynamics naturally produce a large-scale electromagnetic field which subsequently evolves through the reheating phase. Instead of going into the details of the magnetogenesis mechanism, we concentrate on dynamics during reheating considering various inflationary models. In our analysis, we assume the negligible Schwinger effect on magnetogenesis. The paper is arranged as follows: in Sec. II, we discuss the $I^{2} F F$ model of inflationary magnetogenesis and shown how Faraday's induction effect impacts the present-day magnetic field strength. In Sec. II, we discuss in detail the methodology of probing the reheating phase through the present-day large-scale magnetic field combined with CMB anisotropy. In Sec. III, we derive an approximate analytical expression that relates the magnetic power spectrum in the present universe with reheating parameters. In Sec. IV, we finally show how our analysis constrains the reheating and magnetogenesis model considering few observationally viable inflationary scenarios.

\section{INFLATIONARY MAGNETOGENESIS: GENERAL DISCUSSION}

During inflation, the large-scale magnetic field is generated out of the quantum vacuum and then subsequently evolves through various phases of our universe. Therefore, the evolving magnetic field must encode valuable information about the reheating. Considering the present value of the large-scale magnetic field, we can place constraints not only on the parameters of the reheating phase but also on the magnetogenesis model itself. Contrary to the 
convention, the crucial point of our present analysis is the assumption of conductivity being negligible until the end of reheating. The reason being the universe turns into the plasma state nearly at the end of reheating. From the large number of studies [12-14,56], it is observed that almost the entire period of reheating is primarily dominated by inflaton. In the context of the inflationary magnetogenesis scenario, this particular assumption has recently been proposed to be important [18] during reheating. Conventionally after the end of inflation, the magnetic field on the superhorizon scales is assumed to be redshifted with respect to the scale factor $a$ as $B^{2} \propto 1 / a^{4}$ provided inflaton energy density transfers into plasma instantaneously, and the universe becomes a good conductor instantly right after the end of inflation. Hence, the electric field ceases to exist. However, in Ref. [18] it has been shown that if the conductivity remains small, redshifts of magnetic energy density becomes slower, $B^{2} \propto 1 / a^{6} H^{2}$, due to electromagnetic Faraday induction. Here $H$ is the Hubble parameter. This helps one to obtain the required value of the present-day large-scale magnetic field considering the large class inflationary model, which we describe below.

\section{A. Quantizing the Ratra model: Electromagnetic power spectrum}

The simplest magnetogenesis scenario we will be considering here is the known as the Ratra model [20] with the interaction Lagrangian $I(\phi)^{2} F F$. In this interacting Lagrangian, the conformal symmetry is explicitly broken by the scalar field coupling function $I(\phi)$. During inflation, the model generally predicts a strong primordial electric field than the magnetic field, and that can backreact to invalidate the mechanism itself. We discuss the model in detail and show how the reheating phase can cure this backreaction problem. In the frame of a comoving observer having four-velocity $u^{\mu}\left(u^{i}=0, u_{\mu} u^{\mu}=-1\right)$, the magnetic and electric fields are defined as

$$
E_{\mu}=u^{\nu} F_{\mu \nu}, \quad B_{\mu}=\frac{1}{2} \epsilon_{\mu \nu \rho \sigma} u^{\sigma} F^{\nu \rho} .
$$

Where, $F_{\mu \nu}=\partial_{\mu} A_{\nu}-\partial_{\nu} A_{\mu}$ is the electromagnetic field tensor and $\epsilon_{\mu \nu \rho \sigma}$ is a totally antisymmetric tensor. The background is the well known FLRW metric with the time-dependent scale factor $a(\tau)$ expressed in conformal coordinate,

$$
d s^{2}=a(\tau)^{2}\left(-d \tau^{2}+d \mathbf{x}^{2}\right) .
$$

As already described before, the gauge field action is taken to be,

$$
S=-\frac{1}{4} \int d^{4} x \sqrt{-g} I(\tau)^{2} F_{\mu \nu} F^{\mu \nu} .
$$

At this point, let us point out that one can consider other inflationary magnetogeneis models with axion-electromagnetic field coupling [57-59], higher curvature coupling $[60,61]$ and apply our methodology presented here to not only constraint the reheating phase but also make the models under consideration viable.

In the inflationary magnetogenesis scenario, the essential idea is to quantize the electromagnetic field in the classical inflationary background. Here $I(\tau)^{2} \equiv I(\phi(\tau))$, therefore, is the time-dependent coupling arising from some classical background scalar field. To maintain generality, we do not specify any background dynamics of the scalar field. Through this coupling, the electromagnetic field experiences the spatially flat expanding FLRW background. To quantize the field components, $A_{\mu}$ are expressed in terms of irreducible scalar and vector components as follows,

$$
A_{\mu}=\left(A_{0}, \partial_{i} S+v_{i}\right) \quad \text { with } \quad \partial_{i} V_{i}=0 .
$$

In the standard canonical quantization procedure, one writes $V_{i}$ in terms of the annihilation $\left(a_{k}\right)$ and the creation operator $\left(a_{k}^{\dagger}\right)$ as

$$
\begin{aligned}
V_{i}(\tau, x)= & \sum_{p=1,2} \int \frac{d^{3} k}{(2 \pi)^{3}} \epsilon_{i}^{(p)}(\mathbf{k})\left\{e^{i \mathbf{k} \cdot \mathbf{x}} a_{k}^{(p)} u_{k}^{(p)}(\tau)\right. \\
& \left.+e^{-i \mathbf{k} \cdot \mathbf{x}} a_{k}^{\dagger(p)} u_{k}^{*(p)}(\tau)\right\},
\end{aligned}
$$

here the $\epsilon_{i}^{(p)}(k)$ is the polarization vector corresponding to the two polarization direction $p=1,2$, which satisfy the following relations, $\epsilon_{i}^{(p)}(\mathbf{k}) k_{i}=0, \epsilon_{i}^{(p)}(\mathbf{k}) \epsilon_{i}^{(q)}(\mathbf{k})=\delta_{p q}$. The creation and annihilation operators in Eq. (5) namely $a_{k}^{(p)}$ and $a_{k}^{\dagger(p)}$ are time independent. They satisfy the commutation relation

$$
\begin{aligned}
{\left[a_{k}^{(p)}, a_{h}^{(q)}\right] } & =\left[a_{k}^{\dagger(p)}, a_{h}^{\dagger(q)}\right]=0, \\
{\left[a_{k}^{(p)}, a_{h}^{\dagger(q)}\right] } & =(2 \pi)^{3} \delta^{p q} \delta^{(3)}(\mathbf{k}-\mathbf{h}) .
\end{aligned}
$$

All the dynamics of the field will be encoded into the mode function which satisfy the following equation of motion,

$$
u_{k}^{(p) \prime \prime}+2 \frac{I^{\prime}}{I}+k^{2} u_{k}^{(p) \prime}=0 .
$$

Where the prime denotes derivative with respect to the proper time $\tau$. Conventionally the electromagnetic power spectrum is expressed in terms of those mode functions as follows,

$$
P_{E}(k)=\frac{k^{3}}{2 \pi^{2} a^{4}} \sum_{p=1,2}\left|u_{k}^{(p) \prime}\right|^{2} ; \quad P_{B}(k)=\frac{k^{5}}{2 \pi^{2} a^{4}} \sum_{p=1,2}\left|u_{k}^{(p)}\right|^{2} .
$$


However, an elegant way to express the above power spectrum is in terms of the Bogliubov coefficient which has a direct physical interpretation in terms of quantum particle production. For this Hamiltonian is written in terms of time independent creation and annihilation operator as follows,

$$
\begin{aligned}
H= & \frac{I^{2}(\tau)}{2} \sum_{p=1,2}\left(k\left|u_{k}^{(p)}\right|^{2}+\frac{1}{k}\left|u_{k}^{(p)}\right|^{2}\right)\left(a_{k}^{(p) \dagger} a_{k}^{(p)}+a_{-k}^{(p)} a_{-k}^{(p) \dagger}\right) \\
& +\left(k\left(u_{k}^{(p)}\right)^{2}+\frac{1}{k}\left(u_{k}^{(p)}\right)^{2}\right) a_{k}^{(p)} a_{-k}^{(p)} \\
& +\left(k\left(u_{k}^{(p) *}\right)^{2}+\frac{1}{k}\left(u_{k}^{*(p)}\right)^{2}\right) a_{-k}^{(p) \dagger} a_{k}^{(p) \dagger} \\
& +\frac{1}{2}\left(a_{k}^{(p) \dagger} a_{k}^{(p)}-a_{-k}^{(p)} a_{-k}^{(p) \dagger}\right) .
\end{aligned}
$$

This is not diagonal. Therefore, to diagonalize, we employ the Bogoliubov transformation. In this transformation, new set of time dependent creation and annihilation operators $\left(b_{k}^{(p)}(\tau), b_{k}^{(p) \dagger}(\tau)\right)$ are defined in term of old ones. And the new basis of the Hilbert space so constructed diagonalizes the above Hamiltonian,

$$
\begin{aligned}
b_{k}^{(p)}(\tau) & =\alpha_{k}^{(p)}(\tau) a_{k}^{(p)}+\beta_{k}^{(p) *} a_{-k}^{(p) \dagger}, \\
b_{k}^{(p) \dagger}(\tau) & =\alpha_{k}^{(p) *}(\tau) a_{k}^{(p) \dagger}+\beta_{k}^{(p)}(\tau) a_{-k}^{(p)} .
\end{aligned}
$$

Where $\alpha_{k}^{(p)}$ and $\beta_{k}^{(p)}$ are the Bogoliubov coefficients defined as

$$
\begin{aligned}
& \alpha_{k}^{(p)}(\tau)=I\left(\sqrt{\frac{k}{2}} u_{k}^{(p)}+\frac{i}{\sqrt{2 k}} u_{k}^{\prime(p)}\right) \\
& \beta_{k}^{(p)}(\tau)=I\left(\sqrt{\frac{k}{2}} u_{k}^{(p)}-\frac{i}{\sqrt{2 k}} u_{k}^{\prime(p)}\right) .
\end{aligned}
$$

The Bogoliubov coefficients follow the following normalization condition

$$
\left|\alpha_{k}^{(p)}\right|^{2}-\left|\beta_{k}^{(p)}\right|^{2}=1
$$

With all these ingredients one represents the power spectrum in terms of Bogoliubov coefficients, $\alpha_{k}^{(p)}$ and $\beta_{k}^{(p)}$ as follows,

$$
\begin{aligned}
& \mathcal{P}_{E}(k)=\frac{k^{4}}{4 \pi^{2} a^{4} I^{2}} \sum_{p=1,2}\left|\alpha_{k}^{(p)}-\beta_{k}^{(p)}\right|^{2} ; \\
& \mathcal{P}_{B}(k)=\frac{k^{4}}{4 \pi^{2} a^{4} I^{2}} \sum_{p=1,2}\left|\alpha_{k}^{(p)}+\beta_{k}^{(p)}\right|^{2}
\end{aligned}
$$

Considering the superhorizon limit, the above expressions for the electromagnetic power spectrum can be further simplified by extracting the amplitude and phase of those coefficients. By using the normalization condition Eq. (13) one can write,

$$
\begin{aligned}
\left|\alpha_{k}^{(p)} \pm \beta_{k}^{(p)}\right|^{2}= & 1+2\left|\beta_{k}^{(p)}\right|^{2} \\
& \pm\left|\beta_{k}^{(p)}\right| \sqrt{1+\left|\beta_{k}^{(p)}\right|^{2}} \cos \left\{\arg \left(\alpha_{k}^{(p)} \beta_{k}^{*(p)}\right)\right\}
\end{aligned}
$$

Where, the phase factor is expressed as $\arg \left(\alpha_{k}^{(p)} \beta_{k}^{*(p)}\right) \equiv$ $\pi+\theta_{k}^{(p)}$. In the following discussion, we consider a specific model of the electromagnetic coupling function.

\section{Magnetogenesis: Modeling the coupling function}

In order to study further we consider the following widely considered power law form of the coupling function [20]

$$
I(\tau)= \begin{cases}\left(\frac{a_{\mathrm{end}}}{a}\right)^{n} & a \leq a_{\mathrm{end}} \\ 1 & a \geq a_{\mathrm{end}},\end{cases}
$$

where $a_{\text {end }}$ is the scale factor at the end of inflation. At this stage, let us point out that, in all the previous analyses, $n$ has been considered to be an integer. For generality, we assume $n$ to be arbitrary. In the end, we want to constrain its value through observation. Furthermore, after the end of inflation, the function form of $I(\phi)$ will be chosen in such a way that the usual conformal electrodynamics is restored.

Our main interest is to understand the large-scale magnetic field assumed to be produced during the initial stage of inflation. Therefore, throughout our analysis, we assume the Hubble parameter to be constant. This also helps us to give a clear picture in terms of analytic solutions. The perfect de-Sitter background with Hubble parameter $H_{\text {inf }}$, one obtains the solution for the mode function as,

$$
u_{k}=\frac{1}{2 I}\left(\frac{\pi}{a H_{\mathrm{inf}}}\right)^{\frac{1}{2}} H_{-n+\frac{1}{2}}^{(1)}\left(\frac{k}{a H_{\mathrm{inf}}}\right)
$$

which leads to Bunch-Davies vacuum state at the subHorizon scale. $H_{-n+\frac{1}{2}}^{(1)}\left(\frac{k}{a H_{\text {inf }}}\right)$ is the Hankel function of the first kind. The Hankel functions are defined in terms of the Bessel function of first and second (Neumann) kind.

$$
H_{\nu}\left(\frac{k}{a H_{\text {inf }}}\right)=J_{\nu}\left(\frac{k}{a H_{\text {inf }}}\right)+i Y_{\nu}\left(\frac{k}{a H_{\text {inf }}}\right)
$$

Here $\nu=-n \pm \frac{1}{2}$. Defining a new variable $z=k / a H_{\text {inf }}$, the time dependent Bogoliubov coefficient are expressed as, 


$$
\begin{aligned}
& \alpha_{k}=\left(\frac{\pi z}{8}\right)^{\frac{1}{2}}\left\{H_{-n+\frac{1}{2}}^{(1)}(z)-i H_{-n-\frac{1}{2}}^{(1)}(z)\right\} ; \\
& \beta_{k}=\left(\frac{\pi z}{8}\right)^{\frac{1}{2}}\left\{H_{-n+\frac{1}{2}}^{(1)}(z)+i H_{-n-\frac{1}{2}}^{(1)}(z)\right\}
\end{aligned}
$$

As we have already described before, our focus is to understand the reheating constraints on inflationary and magnetogenesis models considering the CMB anisotropy and LSMF field, which are naturally classified as largescale observables. Our discussion will be mainly in the superhorizon limit. Therefore, in this limit following condition will be satisfied

$$
\frac{1}{\left|\beta_{k}^{(p)}\right|^{2}} \ll \theta_{k}^{(p)} \ll 1
$$

This essentially suggests that any mode starting from the Bunch-Davis vacuum at the subhorizon scale transforms into a highly squeezed state, parametrized by $\left|\beta_{k}^{(p)}\right|^{2} \gg 1$, after its horizon exit during inflation. Using this condition, one gets the following simplified form of the electromagnetic power spectrum,

$$
\begin{aligned}
& \mathcal{P}_{E}(k) \simeq \frac{k^{4}}{4 \pi^{2} a^{4} I^{2}} \sum_{p=1,2} 4\left|\beta_{k}^{(p)}\right|^{2} \\
& \mathcal{P}_{B}(k) \simeq \frac{k^{4}}{4 \pi^{2} a^{4} I^{2}} \sum_{p=1,2}\left|\beta_{k}^{(p)}\right|^{2}\left(\theta_{k}^{(p)}\right)^{2}
\end{aligned}
$$

For arbitrary value of $n$, the final expression for the spectrum assume the follow forms,

$$
\begin{aligned}
\mathcal{P}_{E}(k) \simeq & \frac{4 k^{4}}{2 \pi^{2} a^{4} I^{2}}\left(\frac{\pi z}{8}\right)\left\{H_{-n+\frac{1}{2}}^{*(1)}(z) H_{-n+\frac{1}{2}}^{(1)}(z)+i H_{-n+\frac{1}{2}}^{*(1)}(z) H_{-n-\frac{1}{2}}^{(1)}(z)\right\} \\
& -\frac{4 k^{4}}{2 \pi^{2} a^{4} I^{2}}\left(\frac{\pi z}{8}\right)\left\{i H_{-n-\frac{1}{2}}^{*(1)}(z) H_{-n+\frac{1}{2}}^{(1)}(z)-H_{-n-\frac{1}{2}}^{(1)}(z) H_{-n-\frac{1}{2}}^{*(1)}(z)\right\} \\
\mathcal{P}_{B}(k) \simeq & \frac{k^{4}}{2 \pi^{2} a^{4} I^{2}}\left(\frac{\pi z}{8}\right)\left\{H_{-n+\frac{1}{2}}^{*(1)}(z) H_{-n+\frac{1}{2}}^{(1)}(z)+i H_{-n+\frac{1}{2}}^{*(1)}(z) H_{-n-\frac{1}{2}}^{(1)}(z)\right\} \\
& -\frac{k^{4}}{2 \pi^{2} a^{4} I^{2}}\left(\frac{\pi z}{8}\right)\left\{i H_{-n-\frac{1}{2}}^{*(1)}(z) H_{-n+\frac{1}{2}}^{(1)}(z)-H_{-n-\frac{1}{2}}^{(1)}(z) H_{-n-\frac{1}{2}}^{*(1)}(z)\right\} \\
& \times\left\{\arg \left[\frac{\pi z}{8}\left(H_{-n+\frac{1}{2}}^{(1)}(z)-i H_{-n-\frac{1}{2}}^{(1)}(z)\right)\left(H_{-n+\frac{1}{2}}^{*(1)}(z)-i H_{-n-\frac{1}{2}}^{*(1)}(z)\right)\right]-\pi\right\}^{2}
\end{aligned}
$$

In our final numerical analysis, we will be considering this expression. To this end, it is important to remind the reader, the widely studied case with positive integer value o $n$ which leads to the following simplified form of the electromagnetic power spectra at the superhorizon scale $\left(k \ll a H_{\text {inf }}\right)$ as [18]

$$
\mathcal{P}_{E}(k) \simeq \frac{8 \Gamma\left(n+\frac{1}{2}\right)^{2} H_{\mathrm{inf}}^{4}}{I^{2} \pi^{3}}\left(\frac{k}{2 a H_{\mathrm{inf}}}\right)^{-2(n-2)} ; \quad \mathcal{P}_{B}(k) \simeq \frac{8 \Gamma\left(n-\frac{1}{2}\right)^{2} H_{\mathrm{inf}}^{4}}{I^{2} \pi^{3}}\left(\frac{k}{2 a H_{\mathrm{inf}}}\right)^{-2(n-3)}
$$

With this expression, subsequent magnetic field evolution has been widely studied, considering the magnetic energy density decreasing as $|B|^{2} \sim 1 / a^{4}$.

\section{After inflation dynamics: Reheating}

In order to associate the observed current magnetic field with the magnetic field produced during inflation, it is essential to study the subsequent evolution. Most of the studies so far considered the fact that when $I^{2}$ becomes constant at the end of the inflation, the comoving photon density $\left|\beta_{k}^{(p)}\right|^{2}$ is conserved. Consequently, the magnetic power redshifts as $\mathcal{P}_{B}(k) \propto a^{-4}$ until today. Before embarking on our proposal, let us briefly discuss the widely studied case for the sake of completeness. Thus, at the end of the inflation, the phase parameter $\left(\theta_{k}^{\text {end }}\right)$ and the photon density $\left(\left|\beta_{k}^{\text {end }}\right|^{2}\right)$ are identified as

$$
\begin{gathered}
\theta_{k}^{\text {end }}=\left\{\operatorname{Arg}\left[\alpha_{k}\left(z_{\text {end }}\right) \beta_{k}^{*}\left(z_{\text {end }}\right)\right]-\pi\right\}, \\
\left|\beta_{k}^{\text {end }}\right|^{2}=\left(\frac{\pi z_{\text {end }}}{8}\right)\left\{H_{-n+\frac{1}{2}}^{*(1)}\left(z_{\text {end }}\right) H_{-n+\frac{1}{2}}^{(1)}\left(z_{\text {end }}\right)+i H_{-n+\frac{1}{2}}^{*(1)}\left(z_{\text {end }}\right) H_{-n-\frac{1}{2}}^{(1)}\left(z_{\text {end }}\right)\right\} \\
-\left(\frac{\pi z_{\text {end }}}{8}\right)\left\{i H_{-n-\frac{1}{2}}^{*(1)}\left(z_{\text {end }}\right) H_{-n+\frac{1}{2}}^{(1)}\left(z_{\text {end }}\right)-H_{-n-\frac{1}{2}}^{(1)}\left(z_{\text {end }}\right) H_{-n-\frac{1}{2}}^{*(1)}\left(z_{\text {end }}\right)\right\} .
\end{gathered}
$$


Here we define $z_{\text {end }} \equiv k / a_{\text {end }} H_{\text {inf }}$. For any arbitrary values of $n$, the magnetic power spectrum is

$$
\mathcal{P}_{B}^{\text {end }}(k) \simeq \frac{k^{4}}{2 \pi^{2} a_{\text {end }}^{4}}\left(\theta_{k}^{\text {end }}\right)^{2}\left|\beta_{k}^{\text {end }}\right|^{2} .
$$

For positive integer $n$, the expression above (27) boils down to

$$
\mathcal{P}_{B}^{\text {end }}(k) \simeq \frac{8 \Gamma\left(n-\frac{1}{2}\right)^{2}}{\pi^{3}} H_{\text {Inf }}^{4}\left(\frac{k}{2 a_{\text {end }} H_{\text {Inf }}}\right)^{-2(n-3)},
$$

at the superhorizon scale $k \ll a H_{\text {inf }}$. Considering nontrivial dynamics, the magnetic power spectrum at the present universe can be correlated with the magnetic power at the end of the inflation through the following standard equation

$$
\mathcal{P}_{B 0}(k) \simeq \mathcal{P}_{B}^{\text {end }}(k)\left(\frac{a_{\text {end }}}{a_{0}}\right)^{4},
$$

where $a_{\text {end }} / a_{0}$ can be expressed in terms of inflationary $e$-folding number $\left(N_{k}\right)$ as

$$
\frac{a_{\mathrm{end}}}{a_{0}}=\frac{a_{\mathrm{end}}}{a_{k}} \frac{k^{\prime}}{a_{0} H_{\mathrm{Inf}}}=\left(\frac{k^{\prime}}{a_{0}}\right) \frac{e^{N_{k}}}{H_{\mathrm{Inf}}} .
$$

Here $k^{\prime} / a_{0}=0.05 M_{p c}^{-1}$ is taken as pivot scale set by Planck observation. By combining equations (28), (29), and (30), the expression for the magnetic power considering integer values of $n$, follow the equation

$$
\mathcal{P}_{B 0} \simeq \frac{\Gamma\left(n-\frac{1}{2}\right)^{2}}{2^{3-2 n} \pi^{3}}\left(\frac{k}{a_{0}}\right)^{6-2 n}\left(\frac{k^{\prime}}{a_{0}}\right)^{2 n-2} e^{2(n-1) N_{k}} .
$$

It has already been observed and also shown in Fig. 1 the well-known fact that magnetic strength of order $10^{-9}$ $10^{-22}$ Gauss on $1 \mathrm{Mpc}$ scales $[21,36,62,63]$ cannot be obtained by the above magnetic power spectrum for high-scale inflation model such as Higgs-Starobinsky inflation. Several models have been constructed to avoid this problem without much success related to the theoretical issues which we discuss in the next section. From here itself, we will advocate the importance of considering the effect of reheating more seriously than the model building. In the recent paper [18], authors have shown that considering negligible electrical conductivity during reheating, Faraday's law of electromagnetic induction plays an interesting role in modifying the magnetic field evolution. Therefore, we want to see how this induction effect can safely generate the presently observed magnetic field for different high-scale inflationary models. Consideration of $\mathrm{CMB}$ anisotropy into the analysis further reveals an intricate interconnection among various apparently disconnected cosmological parameters such as $\left(n_{s}, P_{B 0}, T_{r e}, w_{r e}\right)$. Consequently, our analysis opens up an interesting new

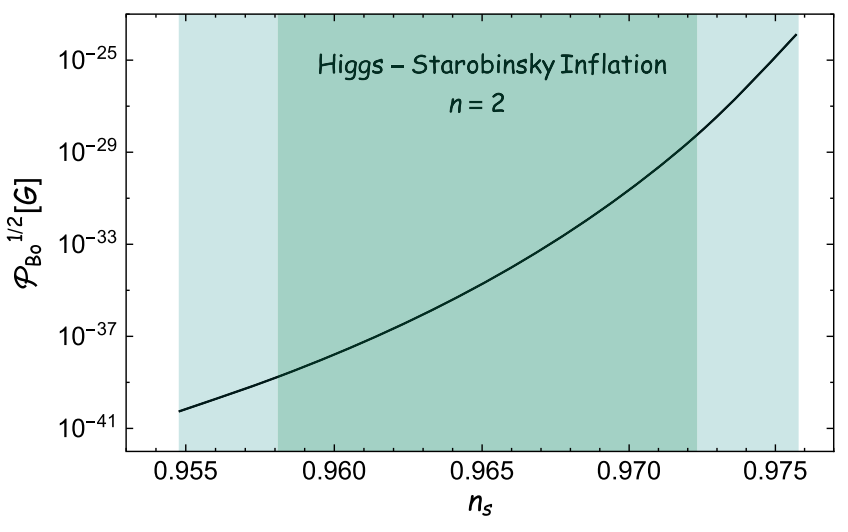

FIG. 1. We plot the variation of the present magnetic field's strength as a function of the spectral index within $2 \sigma$ range of $n_{s}$ from Planck [1] for Higgs-Starobinsky inflationary model. It is clear that the required magnetic field strength $\left(10^{-9} \sim 10^{-22}\right) \mathrm{G}$ is difficult to achieve within the conventional framework, unless one introduces slow decreasing rate of magnetic energy density in some early state of universe evolution.

possibility of probing the reheating dynamics and simultaneously constraining the inflationary and magnetogenesis model parameters through the evolution of the primordial magnetic field, which has not been considered before.

After the end of inflation, the assumption of the gauge kinetic function $I(\phi)$ being unity renders the postinflationary evolution of the electromagnetic field as standard Maxwellian. Therefore, further production of gauge field from the quantum vacuum ceases to exist. The electromagnetic field produced during inflation will cross the horizon and turn into the classical one, which will evolve during this phase. In the Fourier space, the mode function solution of the free Maxwell equation is,

$u_{k}^{(p)}=\frac{1}{\sqrt{2 k}}\left\{\alpha_{k}^{(p)}\left(z_{\text {end }}\right) e^{-i k\left(\tau-\tau_{\text {end }}\right)}+\beta_{k}^{(p)}\left(z_{\text {end }}\right) e^{-i k\left(\tau-\tau_{\text {end }}\right)}\right\}$,

here $\tau_{\text {end }}$ represents the end of inflationary era. For arbitrary value of $n$, the phase factor is calculated to be

$\theta_{k}^{(p)}=\left\{\operatorname{Arg}\left[\alpha_{k}^{(p)}\left(z_{\text {end }}\right) \beta_{k}^{(p) *}\left(z_{\text {end }}\right)\right]-\pi\right\}-2 k\left(\tau-\tau_{\text {end }}\right)$.

Where the elapsed conformal time is obtained as

$$
\tau-\tau_{\mathrm{end}}=\int_{a_{\mathrm{end}}}^{a} \frac{d a}{a^{2} H} .
$$

Since there is no further production of gauge field, the photon number density $\left|\beta_{k}\right|^{2}$ becomes independent of time and follows the same equation (26) as before. However, as emphasized already, the Faraday induction will come into 
play during this phase. To see this, let us first express the magnetic power spectra at the end of reheating parametrized by the scale factor $\left(a_{r e}\right)$ as

$$
\mathcal{P}_{B_{r e}}(k) \simeq \frac{k^{4}}{2 \pi^{2} a_{r e}^{4}}\left(\theta_{k}^{r e}\right)^{2}\left|\beta_{k}^{\text {end }}\right|^{2},
$$

where $\theta_{k}^{r e}$ is the phase parameter at the end of the reheating era, which is defined as,

$\theta_{k}^{r e}=\left\{\operatorname{Arg}\left[\alpha_{k}\left(z_{\mathrm{end}}\right) \beta_{k}^{*}\left(z_{\mathrm{end}}\right)\right]-\pi\right\}-2 k \int_{a_{\mathrm{end}}}^{a_{r e}} \frac{d a}{a^{2} H}$.

Notable term of the above expression is the second one which leads to the nonconventional dynamics of the magnetic field. Assuming the constant equation of state during reheating dynamics the special term boils down to the following simple from,

$$
2 k\left(\tau-\tau_{\text {end }}\right) \rightarrow \frac{4 k}{3 \omega+1}\left(\frac{1}{a H}-\frac{1}{a_{\mathrm{end}} H_{\mathrm{inf}}}\right) .
$$

The phase term contributes to the dynamics of the magnetic power spectrum in two different ways. The conventional one will be associated with the redshift factor $\propto a^{-4}$ emerging from the first term in the right-hand side of the Eq. (33). The important one is associated with the redshift factor $\propto a^{-6} H^{-2}$ emerged out from $k / a H$ term. As the expansion of the universe is decelerating after inflation, the leading contribution to the evolution of the magnetic power would be controlled by the latter one Eq. (33). However, the electric field energy dilutes following the conventional form

$$
\mathcal{P}_{E}(k) \simeq \frac{2 k^{4}}{\pi^{2} a^{4}}\left|\beta_{k}^{\text {end }}\right|^{2} .
$$

For large scale $(k \ll a H)$, the above expression boils down to,

$\mathcal{P}_{E}(k) \simeq \frac{8 \Gamma\left(n+\frac{1}{2}\right)^{2}}{\pi^{3}} H_{\text {inf }}^{4}\left(\frac{k}{2 a_{\text {end }} H_{\text {inf }}}\right)^{-2(n-2)}\left(\frac{a_{\text {end }}}{a}\right)^{4}$,

for integer values of $n$. After the end of reheating, inflaton energy is converted into conducting plasma containing all the standard model particles. Due to large electrical conductivity, the primordial electric field decays to zero, and comoving large-scale magnetic energy density freezes to a constant value until today. Therefore, the final general expression of our interest is the present-day magnetic field strength given as

$$
\mathcal{P}_{B_{0}}(k)=\mathcal{P}_{B_{r e}}(k)\left(\frac{a_{r e}}{a_{0}}\right)^{4} \simeq \frac{k^{4}}{2 \pi^{2}}\left(\theta_{k}^{r e}\right)^{2}\left|\beta_{k}^{\text {end }}\right|^{2} \frac{1}{a_{0}^{4}} .
$$

If we take integer value of $n$, at super horizon scale, the above expression will transforms into [18]

$$
\begin{aligned}
\mathcal{P}_{B 0}(k) \simeq & \frac{8 \Gamma\left(n-\frac{1}{2}\right)^{2}}{\pi^{3}} H_{\mathrm{inf}}^{4}\left(\frac{k}{2 a_{\mathrm{end}} H_{\mathrm{inf}}}\right)^{-2(n-3)}\left(\frac{a_{\mathrm{end}}}{a_{0}}\right)^{4} \\
& \times\left\{1+(2 n-1) \int_{a_{\mathrm{end}}}^{a_{\text {en }}} \frac{d a}{a} \frac{a_{\mathrm{end}} H_{\mathrm{inf}}}{a H}\right\}^{2},
\end{aligned}
$$

where $a_{0}$ is the scale factor at the present time. In the next section we will briefly discuss about standard theoretical issues related to the magnetogenesis model.

\section{a. Discussion on strong coupling and backreaction problem}

In order to generate the large-scale magnetic field of required strength, conventional magnetogenesis models encounter either the strong coupling or backreaction problem. In order to alleviate these issues, several attempts $[19,29,64-77]$ have been made either by advocating different forms of the gauge coupling function or taking nontrivial dynamics of the coupling fields during reheating. In the context of the simplest magnetogenesis model proposed in [20], the gauge kinetic function $I(\tau)$ can essentially be interpreted as time-dependent effective electromagnetic coupling. By considering field-theoretic argument and experimental observations $[29,30]$ it is generically argued that the model either suffers from the strong coupling problem or backreaction problem. In our previous discussion, we have chosen the effective electromagnetic coupling function as a monomial function of the scale factor,

$$
I=\left(\frac{a_{\mathrm{end}}}{a}\right)^{n}=e^{N_{k} n},
$$

where $N_{k}=\ln \left(a / a_{\text {end }}\right)$ associated with a particular scale $k$ is identified as the $e$-folding number during inflation. It is clear from the above expression that once the value of $n$ is chosen to be negative, the gauge kinetic function increases during inflation. Now the behavior of $I(\tau)$ is so chosen that it boils down to unity after inflation. Therefore, during inflation, its magnitude must be less than unity. This is where the origin of the strong coupling problem in the electromagnetic sector lies. Under the field redefinition $A_{\mu} \rightarrow \sqrt{I} A_{\mu}$, the effective electromagnetic coupling $\alpha_{\text {eff }}$, defined through the fermion-gauge field interaction $\mathcal{L}_{\text {int }}=$ $e A_{\mu} J^{\mu}$ modified as

$$
\alpha_{\mathrm{eff}}=\frac{e^{2}}{4 \pi I}=\frac{\alpha}{I}
$$

$e$ is the charge of the fermionic field contributing to the electric current. $\alpha$ is the standard electromagnetic coupling. Nonetheless, as one goes early in the inflationary phase, the effective electromagnetic coupling necessarily becomes very large, which turns the theory nonperturbative. The perturbative computation of magnetogenesis discussed in 
our previous section, will no longer be valid. Keeping this problem aside, if we still follow the analysis as before, large effective electromagnetic coupling $\alpha_{\text {eff }}$, will naturally suppress the gauge field production. This may lead to magnetic field in accord with observed value without any backreaction problem.

On the other hand, if one considers positive values of $n \geq 0$, the whole reasoning expressed just now will be reversed. The gauge kinetic function $I(\tau)$ will be larger than unity, and hence the perturbative magnetogenesis analysis will be perfectly valid for this case. However, considerable reduction of the effective electromagnetic coupling $\alpha_{\text {eff }} \propto 1 / I(\tau)$, specifically during the early inflationary stage of our interest, significantly enhances the production of electromagnetic energy. In such a scenario, the quantum production of electromagnetic energy may take over the background energy density. This is precisely the backreaction problem, which jeopardies the very idea of quantum production from a fixed inflationary background. To avoid the backreaction problem, the energy density of the gauge field must be smaller than the total background energy density $\rho_{\text {tot }}=3 M_{p}^{2} H_{\text {Inf }}^{2}$. For subsequent calculation, let us define the useful parameter which quantifies the amount of background energy density during inflation against the gauge field energy density $\rho_{A}$ as,

$$
\frac{\rho_{A}}{\rho_{\text {tot }}} \leq \zeta
$$

where $\zeta$ is associated with the amplitude of the curvature perturbation measured from CMB anisotropy. The CMB observation constrain the amplitude of the curvature perturbation as $\zeta=4.58 \times 10^{-5}$ [1]. The total gauge field energy density at a given scale factor $a_{T}$ during inflation can be calculated by using Eqs. (14) and (19) as

$$
\begin{aligned}
\rho_{A}\left(a_{T}\right)= & \rho_{E}\left(a_{T}\right)+\rho_{B}\left(a_{T}\right) \\
= & \frac{I\left(a_{T}\right)^{2}}{2} \int_{k_{\mathrm{IR}}}^{k_{T}} \frac{d k}{k}\left\{\mathcal{P}_{E}\left(k, a_{T}\right)+\mathcal{P}_{B}\left(k, a_{T}\right)\right\} \\
= & \frac{I\left(a_{T}\right)^{2}}{2} \int_{k_{\mathrm{IR}}}^{k_{T}} \frac{d k}{4 \pi k H_{\mathrm{inf}}}\left(\frac{k}{a_{T}}\right)^{5}\left\{\left|H_{-n-\frac{1}{2}}^{(1)}\left(\frac{k}{a_{T} H_{\mathrm{inf}}}\right)\right|^{2}\right. \\
& \left.\quad+\left|H_{-n+\frac{1}{2}}^{(1)}\left(\frac{k}{a_{T} H_{\mathrm{inf}}}\right)\right|^{2}\right\} .
\end{aligned}
$$

In the above expression, the $k$-integration ranges from $k_{\mathrm{IR}}$ to $k_{T}$. Associated with our observable universe, the IR cutoff $k_{\mathrm{IR}}$ corresponds to the highest mode that exits the horizon at the beginning of the inflation or approximately the CMB scale, and $k_{T} \sim a_{T} H_{\text {inf }}$ denotes the mode that crosses the horizon at any arbitrary scale $a=a_{T}$. In order to avoid backreaction, the energy density across the scales emerging during inflation $\left(a_{k} \leq a_{T} \leq a_{\text {end }}\right)$, should satisfies the condition (44). So we can set a limit on index $0<n<n_{\max }$ of the gauge coupling function above which the Eq. (44) will be violated. Thus within the aforementioned range of $n$, we can take care of strong coupling and backreaction problems simultaneously.

For example the scale-invariant electric power spectrum which corresponds to $n=2$, the total gauge field energy density at the end of the inflation is

$$
\rho_{A}=\rho_{E}+\rho_{B} \sim \frac{9}{4} \frac{H_{\mathrm{Inf}}^{4}}{\pi^{2}} N_{k}+\frac{H_{\mathrm{Inf}}^{4}}{8 \pi^{2}}\left(1-e^{-2 N_{k}}\right) .
$$

The ratio between the gauge field and the total background energy density is always less than the amplitude of the curvature perturbation $\left(\rho_{A} / \rho_{\text {tot }} \sim 10^{-10}\right)$ for all allowed values of the spectral index. For the scale-invariant electric power spectra model, therefore, the backreaction, as well as the strong coupling problem, can be avoided. However, during the entire inflation period, such models generically produce the magnetic field of insufficient strength. And we will see how the reheating phase helps to enhance the magnitude of the magnetic field to a desirable strength.

Another interesting case is when the generated magnetic field is scale-invariant, which corresponds to $n=3$. However, immediate problem arises in the electric field power spectrum $\mathcal{P}_{E}(k) \propto\left(\frac{k}{a H_{\text {Inf }}}\right)^{-2} \rightarrow \infty$ (see Eq. (24)], which increases rapidly in the large scale limit $\frac{k}{a H_{\text {Inf }}} \rightarrow 0$. Hence electrical energy density exceeds the background inflaton energy density much before the end of the inflation. However, it can be cured by changing the coupling function as follows,

$$
I(\tau)= \begin{cases}\left(\frac{a_{b r}}{a}\right)^{n} & a \leq a_{b r} \\ 1 & a \geq a_{b r}\end{cases}
$$

where $a_{b r}$ is the scale factor defined at a particular point during inflation when

$$
\frac{\rho_{E}\left(a_{b r}\right)+\rho_{B}\left(a_{b r}\right)}{3 M_{p}^{2} H_{\mathrm{Inf}}^{2}}=\zeta .
$$

Therefore, the primary assumption is that the standard maxwell theory is recovered at a point $a_{b r}$ not after the end of inflation. In such a scenario, one can naturally solve the backreaction problem for the $n=3$ magnetogenesis model. However, from the detailed analysis, we found it difficult to obtain the magnetic field within the observable limit. The magnetic field strength turns out to be within $\left(10^{-45}-10^{-50}\right) \mathrm{G}$, which is very small compared with the observational limit. We will discuss this possible scenario in our future work. In our subsequent discussion, we will mainly concentrate on the model with the scale-invariant electric power spectrum during inflation. 


\section{B. Reheating dynamics: Connecting reheating and primordial magnetic field via $\mathrm{CMB}$}

By now, it becomes clear that the primary importance of the reheating phase is to enhance the strength of the largescale magnetic field to the required order. We understood the fact that Faraday's law of electromagnetic induction plays a crucial role in this regard. Therefore, to obtain the correct order of the current magnetic field, understanding the reheating dynamics as well as the evolution of the magnetic field during this period will be of utmost importance. To do that, we will consider two possible reheating models and compare the result.

\section{Case I:}

For this, we follow the effective one fluid description of reheating dynamics proposed in [12], where inflaton energy is assumed to be converted into radiation instantaneously at the end of reheating. The dynamics is parametrized by an effective equation of state $\omega_{\text {eff }}$, reheating temperature $T_{r e}$ and duration $N_{r e}$ ( $e$-folding number during reheating era). In this reheating model, following the approximation as mentioned earlier, one can easily derive the expression for $T_{r e}$ and $N_{r e}$ in terms of inflationary parameters as [56]

$$
\begin{gathered}
T_{r e}=\left(\frac{43}{11 g_{s, r e}}\right)^{\frac{1}{3}}\left(\frac{a_{0} T_{0}}{k^{\prime}}\right) H_{k} e^{-N_{k}} e^{-N_{r e},} \\
N_{r e}=\frac{4}{\left(1-3 \omega_{\text {eff }}\right)}\left[-\frac{1}{4} \ln \left(\frac{45}{\pi^{2} g_{r e}}\right)-\frac{1}{3} \ln \left(\frac{11 g_{s, r e}}{43}\right)\right. \\
\left.-\ln \left(\frac{k^{\prime}}{a_{0} T_{0}}\right)-\ln \left(\frac{V_{\text {end }}^{1 / 4}}{H_{k}}\right)-N_{k}\right],
\end{gathered}
$$

where the present $\mathrm{CMB}$ temperature $T_{0}=2.725 \mathrm{~K}$, the pivot scale $k^{\prime} / a_{0}=0.05 M_{p c}^{-1} . a_{0}$ is the present cosmological scale factor. For simplicity we assume the values of the entropic degrees of freedom $\left(g_{s, r e}\right)$, and the relativistic thermalizing degrees of freedom $\left(g_{r e}\right)$ to be same $g_{s, r e}=g_{r e} \approx 100$. The Eqs. (49) and (50), clearly show the relation among inflationary and reheating parameters. Considering simple canonical inflation potential $V(\phi)$, the inflation model-dependent input parameters, the inflationary $e$-folding number $\left(N_{k}\right)$, and the inflationary Hubble constant $\left(H_{k}\right)$ for a particular CMB scale $k$ are known to be written as

$N_{k}=\log \left(\frac{a_{\mathrm{end}}}{a_{k}}\right)=\int_{\phi_{k}}^{\phi_{\mathrm{end}}} \frac{|d \phi|}{\sqrt{2 \epsilon_{v}} M_{p}}, \quad H_{k}=\frac{\pi M_{p} \sqrt{r_{k} A_{s}}}{\sqrt{2}}$,

here the inflaton field $\phi_{\text {end }}$ defined at the end of the inflation. Furthermore, the scalar spectral index $n_{s}^{k}$, and tensor to scalar ratio $r_{k}$ can be related with the above inflationary parameters $\left(H_{k}, N_{k}\right)$ through the following equations

$$
n_{s}^{k}=1-6 \epsilon\left(\phi_{k}\right)+2 \eta\left(\phi_{k}\right), \quad r_{k}=16 \epsilon\left(\phi_{k}\right) .
$$

Here the slow-roll parameters expressed as

$$
\epsilon_{v}=\frac{M_{p}^{2}}{2}\left(\frac{V^{\prime}}{V}\right)^{2} ; \quad\left|\eta_{v}\right|=M_{p}^{2} \frac{\left|V^{\prime \prime}\right|}{V} .
$$

The above expressions manage to write reheating parameters in terms of the scalar spectral index $\left(n_{s}^{k}\right)$ for a given CMB scale $k$. After identifying all the required parameters, we will now be able to connect CMB and reheating through inflation.

a. Reheating parameters and primordial magnetic field:

Since electrical conductivity during entire period reheating is assumed to negligible, the electric field continues to exist in the post-inflationary era until the universe becomes a perfect conductor. This is the nonzero electric field during reheating, whose dynamics will significantly change the dynamics of the magnetic field and produce a strong magnetic field today.

For this present reheating model, the phase parameter at the end of the reheating $\theta_{k}^{r e}$ in Eq. (36) can be defined as,

$$
\begin{aligned}
\theta_{k}^{r e}= & \left\{\operatorname{Arg}\left[\alpha_{k}\left(z_{\text {end }}\right) \beta_{k}^{*}\left(z_{\text {end }}\right)\right]-\pi\right\} \\
& -\frac{4}{3 \omega_{\text {eff }}+1}\left(\frac{k}{a_{r e} H_{r e}}-\frac{k}{a_{\text {end }} H_{\text {Inf }}}\right),
\end{aligned}
$$

where $H_{r e}$ is the Hubble rate at the end point of reheating. Using evolution of effective density $\rho \propto a^{-3\left(1+\omega_{\text {eff }}\right)}$, one can extract the information about the Hubble parameter at the end of reheating $\left(H_{r e}\right)$ as

$$
H_{r e}=H_{\mathrm{inf}} A_{r e}^{-\frac{3}{2}\left(1+\omega_{\mathrm{eff}}\right)}
$$

with the normalized scale factor $A_{r e}=a_{r e} / a_{\mathrm{end}}=e^{N_{r e}}$. For integer values of $n$, the earlier expression of the phase parameter turns out as

$\theta_{k}^{r e} \simeq \frac{2}{2 n-1} \frac{k}{a_{\mathrm{end}} H_{\mathrm{inf}}}\left\{1+\frac{4 n-2}{3 \omega_{\mathrm{eff}}+1}\left(\frac{a_{\mathrm{end}} H_{\mathrm{inf}}}{a_{r e} H_{r e}}-1\right)\right\}$.

Furthermore, utilizing Eqs. (56) and (35), one can obtain the magnetic power spectrum during the reheating epoch as,

$$
\begin{aligned}
\mathcal{P}_{B_{r e}}(k) \simeq & \frac{8 \Gamma\left(n-\frac{1}{2}\right)^{2}}{\pi^{3} H_{\mathrm{Inf}}^{-4}}\left(\frac{k}{2 a_{\mathrm{end}} H_{\mathrm{inf}}}\right)^{-2(n-3)}\left(\frac{a_{\mathrm{end}}}{a}\right)^{4} \\
& \times\left\{1+\left(\frac{4 n-2}{3 \omega_{\mathrm{eff}}+1}\right)\left(\frac{a_{\mathrm{end}} H_{\mathrm{inf}}}{a H}-1\right)\right\}^{2} .
\end{aligned}
$$


After reheating, the conductivity of the universe becomes sufficiently large. As a consequence, the electric field dies out very fast, and the magnetic field redshifts as $\mathcal{P}_{B} \propto a^{-4}$ till today. Since the comoving magnetic power spectrum is conserved after reheating, the present-day magnetic field obeys the following relation [18]

$$
\begin{aligned}
\mathcal{P}_{B_{0}}(k) \simeq & \frac{8 \Gamma\left(n-\frac{1}{2}\right)^{2}}{\pi^{3} H_{\mathrm{Inf}}^{-4}}\left(\frac{k}{2 a_{\mathrm{end}} H_{\mathrm{Inf}}}\right)^{-2(n-3)}\left(\frac{a_{\mathrm{end}}}{a_{0}}\right)^{4} \\
& \times\left\{1+\left(\frac{4 n-2}{3 \omega_{\mathrm{eff}}+1}\right)\left(\frac{a_{\mathrm{end}} H_{\mathrm{inf}}}{a_{r e} H_{r e}}-1\right)\right\}^{2},
\end{aligned}
$$

where the ratio between the scale factor $a_{0}$ and $a_{r e}$, considering entropy conservation can be expressed as

$$
\frac{a_{0}}{a_{r e}}=\left(\frac{11 g_{s, r e}}{43}\right)^{\frac{1}{3}} \frac{T_{r e}}{T_{0}} .
$$

From the preceding expression, we observe that the magnetic field's present strength explicitly depends on the reheating, and inflationary parameters. Therefore, this opens up a new possibility probing the early phases of universe, particularly the reheating phase through large scale magnetic field. For a specific value of the spectral index, the $\mathcal{P}_{B_{0}}$ has a one to one correspondence with the effective equation of state of reheating $\omega_{\text {eff }}$ and reheating temperature $T_{r e}$. Therefore, an important conclusion we arrived at that the effective equation of state is no longer a free parameter rather it can be fixed by the present value of $\mathcal{P}_{B_{0}}$ via $C M B$.

\section{Case II:}

In the earlier case, we have not taken into account explicit decay of the inflaton field, and additionally, the effective equation of state was assumed to be constant. In this case, we consider perturbative reheating scenario following the methodology discussed in [14]. Therefore, unlike previous reheating scenario, the effective equation of state is time-dependent for the present case. The average inflaton equation of state is taken to be constant though. For example, inflaton potential $V(\phi) \propto \phi^{p}$ gives rise to average equation of state

$$
\omega_{\phi}=\frac{p-2}{p+2},
$$

considering virial theorem [78] when oscillating around its minimum after the end of inflation. Furthermore, for simplicity we assume inflaton decays into radiation only. The Boltzmann equation for the inflaton energy density $\left(\rho_{\phi}\right)$ and radiation energy density $\left(\rho_{r}\right)$ are,

$$
\begin{gathered}
\Phi^{\prime}+\frac{\sqrt{3} M_{p} \Gamma_{\phi}}{m_{\phi}^{2}}\left(1+w_{\phi}\right) \frac{A^{1 / 2} \Phi}{\frac{\Phi}{A^{3 w} \phi}+\frac{R}{A}}=0, \\
R^{\prime}-\frac{\sqrt{3} M_{p} \Gamma_{\phi}}{m_{\phi}^{2}}\left(1+w_{\phi}\right) \frac{A^{\frac{3\left(1-2 w_{\phi}\right)}{2}} \Phi}{\frac{\Phi}{A^{3 w_{\phi}}}+\frac{R}{A}}=0 .
\end{gathered}
$$

Where the comoving densities in terms of the dimensionless variable are used,

$$
\Phi=\frac{\rho_{\phi} A^{3\left(1+w_{\phi}\right)}}{m_{\phi}^{4}} ; \quad R(t)=\frac{\rho_{R} A^{4}}{m_{\phi}^{4}} .
$$

In the above equations, to increase the stability of the numerical solution, we use the inflaton mass $\left(m_{\phi}\right)$ to define the dimensionless scale factor as $A=a / a_{\text {end }}=a m_{\phi}$. For solving the Boltzmann equation, naturally, the initial conditions will be set at the end of inflation as follows,

$$
\Phi(A=1)=\frac{3}{2} \frac{V_{\text {end }}(\phi)}{m_{\phi}^{4}} ; \quad R(A=1)=0 .
$$

For this mechanism the reheating temperature is identified from the radiation temperature $T_{\text {rad }}$ at the point of $H\left(t_{r e}\right)=\Gamma_{\phi}$, when maximum inflaton energy density transfer into radiation.

$$
T_{r e}=T_{\mathrm{rad}}^{\mathrm{end}}=\left(\frac{30}{\pi^{2} g_{r e}}\right)^{1 / 4} \rho_{R}\left(\Gamma_{\phi}, A_{r e}, n_{s}^{k}\right)^{1 / 4} .
$$

To establish one to one correspondence between $T_{r e}$ and $\Gamma_{\phi}$, we combine the equations (64) and (49). To fixed the values of decay width $\Gamma_{\phi}$ in terms of spectral index $\left(n_{s}\right)$, we use one further condition at the end of the reheating

$$
\begin{aligned}
H\left(A_{r e}\right)^{2} & =\left(\frac{\dot{A}_{r e}}{A_{r e}}\right)^{2} \\
& =\frac{\left.\rho_{\phi}\left(\Gamma_{\phi}, A_{r e}, n_{s}^{k}\right)+\rho_{R}\left(\Gamma_{\phi}, A_{r e}, n_{s}^{k}\right)\right)}{3 M_{p}^{2}}=\Gamma_{\phi}^{2} .
\end{aligned}
$$

a. Connecting reheating and primordial magnetic field:

In order to connect the reheating and primordial magnetic field through the $\mathrm{CMB}$, it is necessary to understand the cosmological evolution of the electromagnetic field during the post-inflationary epoch, especially during reheating, which modifies the present strength of the magnetic power spectrum. As we consider the perturbative decay of the inflaton field during reheating, the phase parameter (36) now explicitly depends on the evolution of the two energy components, $\rho_{\phi}$ and $\rho_{R}$ with time

$$
\begin{aligned}
\theta_{k}^{r e}= & \left\{\operatorname{Arg}\left[\alpha_{k}\left(z_{\mathrm{end}}\right) \beta_{k}^{*}\left(z_{\mathrm{end}}\right)\right]-\pi\right\} \\
& -2 k \int_{a_{\mathrm{end}}}^{a_{r e}} \frac{\sqrt{3} M_{p}}{\sqrt{\rho_{\phi}(a)+\rho_{R}(a)}} \frac{d a}{a^{2}} .
\end{aligned}
$$


Furthermore, for integer values of coupling parameters at the super horizon scale, one can find [18]

$$
\begin{aligned}
\theta_{k}^{r e} \simeq & \frac{2}{2 n-1} \frac{k}{a_{\mathrm{end}} H_{\mathrm{Inf}}} \\
& \times\left\{1+(2 n-1) \int_{a_{\mathrm{end}}}^{a_{r e}} \frac{\sqrt{3} M_{p}}{\sqrt{\rho_{\phi}(a)+\rho_{R}(a)}} \frac{a_{\mathrm{end}} H_{\mathrm{inf}}}{a} \frac{d a}{a}\right\},
\end{aligned}
$$

where the time-dependent density components will be followed from Eq. (61). Thus, after combining Eqs. (41), (59), and (67), one can obtain the magnetic power spectrum in the present universe as

$$
\begin{aligned}
\mathcal{P}_{B 0}(k) \simeq & \frac{\Gamma\left(n-\frac{1}{2}\right)^{2}}{\pi^{3}} \frac{2^{2 n-3}\left(2.6 \times 10^{39}\right)}{\left(6.4 \times 10^{-39}\right)^{2 n-6}} \\
& \times\left(\frac{k}{a_{0}} M_{p c}\right)^{-2(n-3)}\left(\frac{11 g_{s, r e}}{43}\right)^{\frac{2-2 n}{3}}\left(\frac{T_{r e}}{T_{0}}\right)^{2-2 n} \\
& \times\left(\frac{H_{\text {inf }}}{\operatorname{GeV}} \frac{1}{A_{r e}}\right)^{2(n-1)} \\
& \times\left\{1+(2 n-1) \int_{1}^{A_{r e}} \frac{\sqrt{3} M_{p} H_{\text {Inf }}}{\sqrt{\rho_{\phi}(a)+\rho_{R}(a)}} \frac{d A}{A^{2}}\right\}^{2} G^{2} .
\end{aligned}
$$

In the preceding expression, we can clearly see the appearance of the reheating parameters, which are the function of inflationary observables. Therefore, we will be able to put bounds on the reheating as well as inflationary model parameters. As we emphasized before, from the measurement of the CMB anisotropy, our goal of this paper would be to constrain the reheating dynamics through inflationary parameters considering the present strength of the magnetic field $\mathcal{P}_{B_{0}}^{1 / 2}$.

\section{MAGNETIC POWER SPECTRUM IN THE PRESENT UNIVERSE IN TERMS OF REHEATING PARAMETERS: AN ANALYTIC STUDY}

Before employing the numerical analysis, in this section, we present an approximate calculation for the present value of the magnetic power spectrum and estimate its value in terms of reheating parameters $\left(T_{r e}, N_{r e}\right)$. Following our previous work [11], the radiation temperature assumes the following form consider Case II reheating scenario,

$$
T_{\mathrm{rad}} \simeq\left\{\frac{2 \Gamma_{\phi}\left(1+\omega_{\phi}\right)}{5-3 \omega_{\phi}} \frac{3 M_{p}^{2} H_{\mathrm{Inf}}}{\beta A^{4}}\left(A^{\frac{5-3 \omega_{\phi}}{2}}-1\right)\right\}^{\frac{1}{4}},
$$

where $\beta=\pi^{2} g_{r e} / 30$. Further, utilizing the above expression of the radiation temperature, we can calculate the approximate expression of the reheating temperature. Subsequently, at the point of $A_{r e}$ the condition $H\left(A_{r e}\right)=\Gamma_{\phi}$ is satisfied, and one can define the reheating temperature as

$$
T_{r e} \simeq\left(\frac{2 \Gamma_{\phi}\left(1+\omega_{\phi}\right)}{5-3 \omega_{\phi}} \frac{3 M_{p}^{2} H_{\mathrm{inf}}}{\beta A_{r e^{2}}^{\frac{3+3 \omega_{\phi}}{2}}}\right)^{\frac{1}{4}}
$$

Here the decay width $\Gamma_{\phi}$ and the normalized scale factor at the end of reheating $\left(A_{r e}\right)$ can be approximated as,

$$
\begin{aligned}
\Gamma_{\phi} & \simeq \frac{\left(5-3 \omega_{\phi}\right) \beta G^{4} A_{r e}^{\frac{3 \omega_{\phi}-5}{2}}}{6 M_{p}^{2} H_{\mathrm{Inf}}}, \\
G & =\left(\frac{43}{11 g_{s, r e}}\right)^{\frac{1}{3}}\left(\frac{a_{0} T_{0}}{k^{\prime}}\right) H_{k} e^{-N_{k}}, \\
A_{r e} & \simeq\left(\frac{\left(5-3 \omega_{\phi}\right)^{2} \beta G^{4}}{12\left(1+\omega_{\phi}\right)^{2} M_{p}^{2} H_{\mathrm{Inf}}^{2}}\right)^{\frac{1}{1-3 \omega_{\phi}}} .
\end{aligned}
$$

From Eq. (35) it is obvious that during reheating phase, the electromagnetic power spectrum is crucially dependent upon the evolution of the phase $\theta_{k}$, which is giving rise to the induced magnetic field. Importantly after the reheating phase ends large scale magnetic field freezes inside the plasma. Therefore, the hierarchy between the inflationary and reheating energy scale sets the strength of the magnetic field today after the inflation. This naturally leads to interesting constraints on the reheating parameters $\left(T_{r e}, N_{r e}, w_{\text {eff }}\right)$ through the current value of the large-scale magnetic field. Inflaton energy density dominates during the initial period of the reheating phase, which enables one to obtain an approximate solution of the inflaton energy density as

$$
\rho_{\phi}(A)=\rho_{\phi}^{\mathrm{end}} A^{-3\left(1+\omega_{\phi}\right)} e^{-\Gamma_{\phi}\left(t-t_{i}\right)} \simeq \rho_{\phi}^{\mathrm{end}} A^{-3\left(1+\omega_{\phi}\right)} .
$$

This helps us to further obtaining the approximate Hubble parameter as

$$
\begin{aligned}
H(A) & =\sqrt{\frac{\rho_{\phi}(A)+\rho_{R}(A)}{3 M_{p}^{2}}} \simeq \frac{H_{\text {Inf }}}{A^{\frac{3\left(1+\omega_{\phi}\right)}{2}}} \sqrt{e^{-\Gamma_{\phi}\left(t-t_{i}\right)}+\frac{A^{\frac{3\left(1+\omega_{\phi}\right)}{2}}}{\eta}} \\
& \simeq \frac{H_{\text {Inf }}}{A^{\frac{3\left(1+\omega_{\phi}\right)}{2}}} \sqrt{1+\frac{A^{\frac{3\left(1+\omega_{\phi}\right)}{2}}}{\eta}}
\end{aligned}
$$

where $\eta=\frac{\left(5-3 \omega_{\phi}\right) H_{\text {inf }}}{2 \Gamma_{\phi}\left(1+\omega_{\phi}\right)}$. With all the above approximate expressions, one can arrive the following expression of the present magnetic power spectrum 

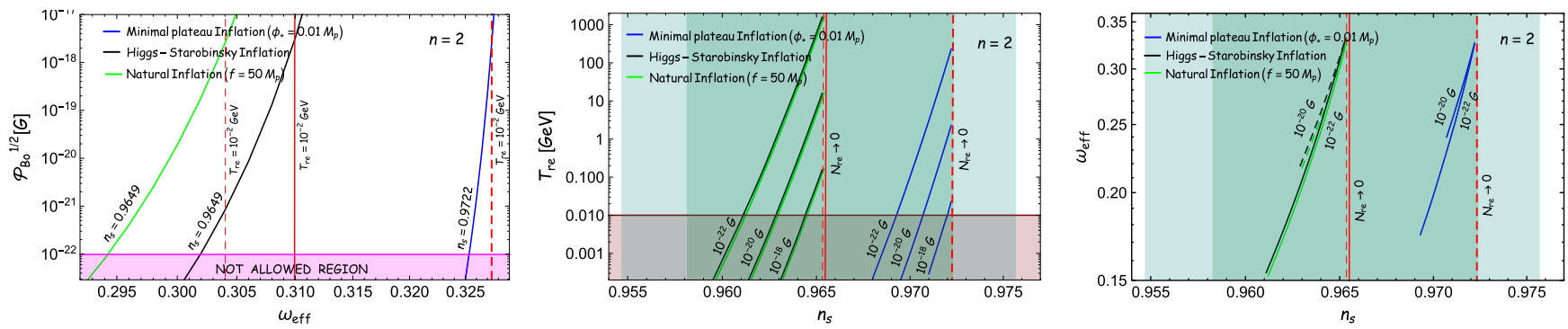

FIG. 2. We plot the magnetic field variation on the upper left side as a function of the effective equation of state for a fixed value of the spectral index. The light pink region indicates the not allowed region as the strength of the magnetic field in that region is less than $10^{-22} \mathrm{G}$ (observed value of the magnetic field on scales $\approx 1 \mathrm{Mpc}$ must be $B_{\text {cosmic }}^{0}>10^{-22} \mathrm{G}$ [21]). The allowed value of the present magnetic field range from $10^{-22} \mathrm{G}$ to the point where the reheating temperature reaches $10^{-2} \mathrm{GeV}$ (comes from BBN constraints), marked as solid and dashed red line. In the middle, we have plotted the variation of reheating temperature as a function of the spectral index for three different values of the current magnetic field $\mathcal{P}_{B_{0}}^{1 / 2}=\left(10^{-18}, 10^{-20}, 10^{-22}\right) \mathrm{G}$. The light brown region indicates the reheating temperature $\left(T_{r e}\right)$ below $10^{-2} \mathrm{GeV}$, which would ruin the predictions of big bang nucleosynthesis (BBN). In the last plot, we have shown the variation of the effective equation of state as a function of the spectral index $\left(n_{s}\right)$ with two different values of $\mathcal{P}_{B_{0}}^{1 / 2}=\left(10^{-20}, 10^{-22}\right) \mathrm{G}$, within the minimum and maximum values of reheating temperature. The deep and light green band indicates $1 \sigma$ and $2 \sigma$ range of $n_{s}$ from Planck [1]. Here we have plotted the results for three different inflationary models (natural inflation, HiggsStarobinsky inflation, minimal plateau model) for the $k$-independent electric power spectrum $(n=2)$.

$$
\begin{aligned}
\mathcal{P}_{B 0}(k) \simeq & \frac{\Gamma\left(n-\frac{1}{2}\right)^{2}}{\pi^{3}} \frac{2^{2 n-3}\left(2.6 \times 10^{39}\right)}{\left(6.4 \times 10^{-39}\right)^{2 n-6}}\left(\frac{k}{a_{0}} M_{p c}\right)^{-2(n-3)} \\
& \times\left(\frac{11 g_{s, r e}}{43}\right)^{\frac{2-2 n}{3}}\left(\frac{T_{r e}}{T_{0}}\right)^{2-2 n}\left(\frac{H_{\mathrm{inf}}}{\mathrm{GeV}} \frac{1}{A_{r e}}\right)^{2(n-1)} \\
& \times\left\{1+(2 n-1)\left[\frac{2}{3 \omega_{\phi}+1}\left(\eta^{\frac{3 \omega_{\phi}+1}{3\left(1+\omega_{\phi}\right)}}-1\right)\right.\right. \\
& \left.\left.+\frac{4}{3 \omega_{\phi}-1} \eta^{1 / 2}\left(A_{r e}^{\frac{3 \omega_{\phi}-1}{4}}-\eta^{\frac{3 \omega_{\phi}-1}{6\left(1+\omega_{\phi}\right)}}\right)\right]\right\}^{2} G^{2} .
\end{aligned}
$$

Interestingly, our analytical expression of the magnetic power spectrum roughly matches with the numerical values.

\section{INFLATION MODELS AND NUMERICAL RESULTS}

The main aim of our study is to see how the present observational limit on the magnitude of large scale magnetic field and the CMB anisotropy can be used to probe the reheating dynamics and put combined constraints on the reheating and inflationary parameter space. We consider different inflationary models and study how the present limits on the magnetic field strength constrain the effective reheating equation of state $\omega_{\text {eff }}$ in terms of the inflationary scalar spectral index $n_{s}$. Further, the allowed range of the current magnetic field can be shown to impose an upper limit on the values of the spectral index $n_{s}^{\max }$ and effective equation of state $\omega_{\mathrm{eff}}^{\max }$, which in turn provides a bound on the maximum possible reheating temperature $T_{r e}^{\max }$. Moreover, to connect reheating parameters such as $\left(T_{r e}\right.$, $\left.\omega_{\text {eff }}\right)$ with the present magnetic field power spectrum $\mathcal{P}_{B_{0}}^{1 / 2}$, we take into account the gauge kinetic function with power $n=2$. We also studied interesting constraints on the maximum possible value of the magnetogenesis model parameter $n=n_{\max }$ and associated maximum reheating temperature $T_{r e}^{\max }$ for different inflationary model.

\section{A. Geneal discussion on our results}

In the conventional study, the magnetic energy density is assumed to be diluted adiabatically with the universe's expansion as $1 / a^{4}$ starting from the end of inflation. This framework never gives rise to enough present-day magnetic field strength for high reheating temperature. In comparison, the high reheating temperature is generically expected in the effective field theory framework. It is in this parlance, dynamics of the magnetic field during reheating should be important to understand. The most important point of our study is the constraints set on the effective reheating equation of state $\omega_{\text {eff }}$. A particular present-day value of $P_{B_{0}}$, the CMB anisotropic constraint and entropy conservation law automatically fixes the reheating equation of state uniquely. Interestingly, this would place a severe restriction on the inflaton potential, which we will study in detail in our subsequent paper. We mainly concentrate on the scale-invariant electric power spectra $(n=2)$ and its evolution for two possible reheating scenarios discussed before. One corresponds to the instantaneous conversion of inflaton energy to radiation at the end of the reheating. The second one is perturbative reheating, where inflaton energy density is transferred into radiation gradually with finite inflaton decay width.

\section{Case I, Instantaneous reheating model:}

Even though we consider the scale-invariant electric field, it is important to remember that it will survive only until the end of reheating. This will condition the magnitude of the large-scale magnetic field in the present-day universe. This intertwined nature of the reheating, 
inflationary parameters, and the large-scale magnetic field is depicted in Fig. 2. From the first panel of Fig. 2, we can clearly predict a unique value of $\omega_{\text {eff }}$ associated with a specific choice of the present magnetic field strength once one fixes the scalar spectral index $n_{s}$. Additionally, for a given $\omega_{\text {eff }}$, the reheating temperature is also determined uniquely. Therefore, taking into account CMB constraints, the reheating phase, conditioned by the negligible electrical conductivity, can be uniquely probed by the evolution of the primordial magnetic field.

In order to have quantitative understanding of our analysis, in the Tables I, III, VI, important reheating parameters such as $\omega_{\text {eff }}$ and $T_{r e}$ for different sample values of the current magnetic field are given for a particular values of the spectral index $n_{s}^{\text {central }}=0.9649$. For any

TABLE I. Probing reheating phase (fixing effective equation of state and reheating temperature). Natural inflation model $\left(f=50 M_{p}\right)$.

\begin{tabular}{lcccc}
\hline \hline & \multicolumn{3}{c}{$\begin{array}{c}\text { Current observed value of the magnetic } \\
\text { field }\left(\mathcal{P}_{B_{0}}^{1 / 2}\right) \text { measured in unit of Gauss }\end{array}$} \\
\cline { 2 - 5 } & & \multicolumn{3}{c}{ Scale invariant electric field $(n=2)$} \\
\cline { 2 - 5 } Parameters & $n_{s}$ & $10^{-18} \mathrm{G}$ & $10^{-20} \mathrm{G}$ & $10^{-22} \mathrm{G}$ \\
\hline$\omega_{\text {eff }}$ & 0.9649 & 0.303 & 0.299 & 0.294 \\
$T_{r e}(\mathrm{GeV})$ & & 0.033 & 3.36 & 339.30 \\
\hline \hline
\end{tabular}

TABLE II. Constraining reheating and inflationary parameters through inflationary magnetogenesis. Natural inflation model $\left(f=50 M_{p}\right)$.

\begin{tabular}{lccc}
\hline \hline & \multicolumn{2}{c}{$\begin{array}{c}\text { Current observed value of the magnetic } \\
\text { field }\left(\mathcal{P}_{B_{0}}^{1 / 2}\right)\end{array}$} \\
\cline { 2 - 4 } & \multicolumn{2}{c}{ Scale invariant electric field $(n=2)$} \\
\cline { 2 - 4 } Parameters & $10^{-18} \mathrm{G}$ & $10^{-20} \mathrm{G}$ & $10^{-22} \mathrm{G}$ \\
\hline$n_{s}^{\min }$ & 0.9645 & 0.9630 & 0.9612 \\
$n_{s}^{\max }$ & 0.9654 & 0.9654 & 0.9654 \\
$\omega_{\mathrm{eff}}^{\min }$ & 0.2842 & 0.2171 & 0.1523 \\
$\omega_{\mathrm{eff}}^{\max }$ & 0.3307 & 0.3303 & 0.3298 \\
$T_{r e}^{\min }(\mathrm{GeV})$ & $10^{-2}$ & $10^{-2}$ & $10^{-2}$ \\
$T_{r e}^{\max }(\mathrm{GeV})$ & 0.16 & 16.42 & $1.6 \times 10^{3}$ \\
\hline \hline
\end{tabular}

TABLE III. Probing reheating phase (fixing effective equation of state and reheating temperature). Higgs-Starobinsky inflation model.

\begin{tabular}{lcccc}
\hline \hline & \multicolumn{2}{c}{$\begin{array}{c}\text { Current observed value of the magnetic field } \\
\left(\mathcal{P}_{B_{0}}^{1 / 2}\right) \text { measured in unit of Gauss }\end{array}$} \\
\cline { 2 - 5 } & & \multicolumn{3}{c}{ Scale invariant electric field $(n=2)$} \\
\cline { 2 - 5 } Parameters & $n_{s}$ & $10^{-18} \mathrm{G}$ & $10^{-20} \mathrm{G}$ & $10^{-22} \mathrm{G}$ \\
\hline$\omega_{\text {eff }}$ & 0.9649 & 0.3093 & 0.3061 & 0.3019 \\
$T_{r e}(\mathrm{GeV})$ & & 0.044 & 4.45 & 447.62 \\
\hline \hline
\end{tabular}

specific choice of the current magnetic field strength within the observational limit, there exists a maximum allowed values of the spectral index $n_{s}^{\max }$ and associated $\omega_{\mathrm{eff}}^{\max }$, which further leads to the maximum permissible value of the reheating temperature shown in the Tables II, IV, VII. We consider three distinct values of $\mathcal{P}_{B_{0}}^{1 / 2}=$

TABLE IV. Constraining reheating and inflationary parameters through inflationary magnetogenesis. Higgs-Starobinsky inflation model.

\begin{tabular}{|c|c|c|c|}
\hline \multirow[b]{3}{*}{ Parameters } & \multirow{2}{*}{\multicolumn{3}{|c|}{$\begin{array}{c}\text { Current observed value of the magnetic field }\left(\mathcal{P}_{B_{0}}^{1 / 2}\right) \\
\text { measured in unit of Gauss } \\
\text { Scale invariant electric field }(n=2)\end{array}$}} \\
\hline & & & \\
\hline & $10^{-18} \mathrm{G}$ & $10^{-20} \mathrm{G}$ & $10^{-22} \mathrm{G}$ \\
\hline$n_{s}^{\min }$ & 0.9644 & 0.9629 & 0.9611 \\
\hline$n_{s}^{\max }$ & 0.9653 & 0.9653 & 0.9653 \\
\hline$\omega_{\mathrm{eff}}^{\min }$ & 0.2852 & 0.2185 & 0.1538 \\
\hline$\omega_{\mathrm{eff}}^{\max }$ & 0.3314 & 0.3311 & 0.3307 \\
\hline$T_{r e}^{\min }(\mathrm{GeV})$ & $10^{-2}$ & $10^{-2}$ & $10^{-2}$ \\
\hline$T_{r e}^{\max }(\mathrm{GeV})$ & 0.16 & 15.7 & $1.6 \times 10^{3}$ \\
\hline
\end{tabular}

TABLE V. Constraining reheating and inflationary parameters through inflationary magnetogenesis (Perturbative reheating dynamics).

\begin{tabular}{lcccc}
\hline \hline & \multicolumn{3}{c}{$\begin{array}{c}\text { Current observed value of the magnetic } \\
\text { field }\left(\mathcal{P}_{B_{0}}^{1 / 2}\right) \text { measured in unit of Gauss }\end{array}$} \\
\cline { 2 - 5 } & \multicolumn{3}{c}{ Scale invariant electric field $(n=2)$} \\
\cline { 2 - 5 } \cline { 5 - 5 } & \multicolumn{2}{c}{$10^{-15} \mathrm{G}$} & \multicolumn{2}{c}{$10^{-18} \mathrm{G}$} \\
\cline { 2 - 5 } Parameters & $(\alpha=1)$ & $\left(\phi_{*}=0.01 M_{p}\right)$ & $(\alpha=1)$ & $\left(\phi_{*}=0.01 M_{p}\right)$ \\
\hline$n_{s}^{\min }$ & 0.966125 & 0.969224 & 0.964075 & 0.96580 \\
$n_{s}^{\max }$ & 0.96905 & 0.9666 & 0.96838 & 0.9700 \\
$\omega_{\phi}^{\min }$ & 0.412 & 0.500 & 0.286 & 0.3100 \\
$\omega_{\phi}^{\max }$ & 0.99 & 0.99 & 0.99 & 0.99 \\
$T_{r e}^{\min }(\mathrm{GeV})$ & $10^{-2}$ & $10^{-2}$ & $10^{-2}$ & $10^{-2}$ \\
$T_{r e}^{\max }(\mathrm{GeV})$ & $3.5 \times 10^{3}$ & 200.1 & $2.32 \times 10^{5}$ & $4.0 \times 10^{3}$ \\
\hline \hline
\end{tabular}

TABLE VI. Probing reheating phase (fixing effective equation of state and reheating temperature). Minimal plateau model $\left(\phi_{*}=0.01 M_{p}\right)$.

\begin{tabular}{lllll}
\hline \hline & \multicolumn{3}{c}{$\begin{array}{c}\text { Current observed value of the magnetic } \\
\text { field }\left(\mathcal{P}_{B_{0}}^{1 / 2}\right) \text { measured in unit of Gauss }\end{array}$} \\
\cline { 2 - 5 } & & \multicolumn{3}{c}{ Scale invariant electric field $(n=2)$} \\
\cline { 2 - 5 } Parameters & $n_{s}$ & $10^{-18} \mathrm{G}$ & $10^{-20} \mathrm{G}$ & $10^{-22} \mathrm{G}$ \\
\hline$\omega_{\text {eff }}$ & 0.9722 & 0.32725 & 0.3264 & 0.3253 \\
$T_{r e}(\mathrm{GeV})$ & & 0.023 & 2.28 & 228.6 \\
\hline \hline
\end{tabular}


TABLE VII. Constraining reheating and inflationary parameters through inflationary magnetogenesis. Minimal plateau model $\left(\phi_{*}=0.01 M_{p}\right)$.

\begin{tabular}{llcc}
\hline \hline & \multicolumn{2}{c}{$\begin{array}{c}\text { Current observed value of the magnetic } \\
\text { field }\left(\mathcal{P}_{B_{0}}^{1 / 2}\right) \text { measured in unit of Gauss }\end{array}$} \\
\cline { 2 - 4 } & \multicolumn{2}{c}{ Scale invariant electric field $(n=2)$} \\
\cline { 2 - 4 } Parameters & $10^{-18} \mathrm{G}$ & $10^{-20} \mathrm{G}$ & $10^{-22} \mathrm{G}$ \\
\hline$n_{s}^{\min }$ & 0.972 & 0.9707 & 0.9693 \\
$n_{s}^{\max }$ & 0.9722 & 0.9722 & 0.9722 \\
$\omega_{\mathrm{eff}}^{\min }$ & 0.3144 & 0.239 & 0.1743 \\
$\omega_{\mathrm{eff}}^{\max }$ & 0.32725 & 0.3264 & 0.3253 \\
$T_{r e}^{\min }(\mathrm{GeV})$ & $10^{-2}$ & $10^{-2}$ & $10^{-2}$ \\
$T_{r e}^{\max }(\mathrm{GeV})$ & 0.023 & 2.3 & 228.6 \\
\hline \hline
\end{tabular}

$\left(10^{-18}, 10^{-20}, 10^{-22}\right) \mathrm{G}$ throughout the discussion. Most importantly, results for the case of instantaneous reheating scenario are the constraints on the effective reheating equation of state $0.15<\omega_{\text {eff }}<0.33$ for quadratic inflaton potential near its minimum. Furthermore, the minimum limiting value of magnetic field $\left(P_{B_{0}}^{1 / 2}<10^{-22}\right) \mathrm{G}$, set an associated maximum reheating temperature around $\sim 1 \mathrm{TeV}$. Therefore, magnetogenesis models with scaleinvariant electric fields appear to be compatible with only the low-scale inflationary model.

\section{Perturbative reheating model (case II):}

The present-day magnetic field and the reheating temperature are intimately connected through the inflaton equation of state, which can be guessed from the previous analysis. However, perturbative reheating is observed to be better suited for understanding the nature of both the inflaton and magnetogenesis model. An important characteristic outcome in considering the perturbative reheating scenario is that the evolution of the large-scale magnetic field permits us to understand the nature of the inflaton field and its observational viability through the constraints on the
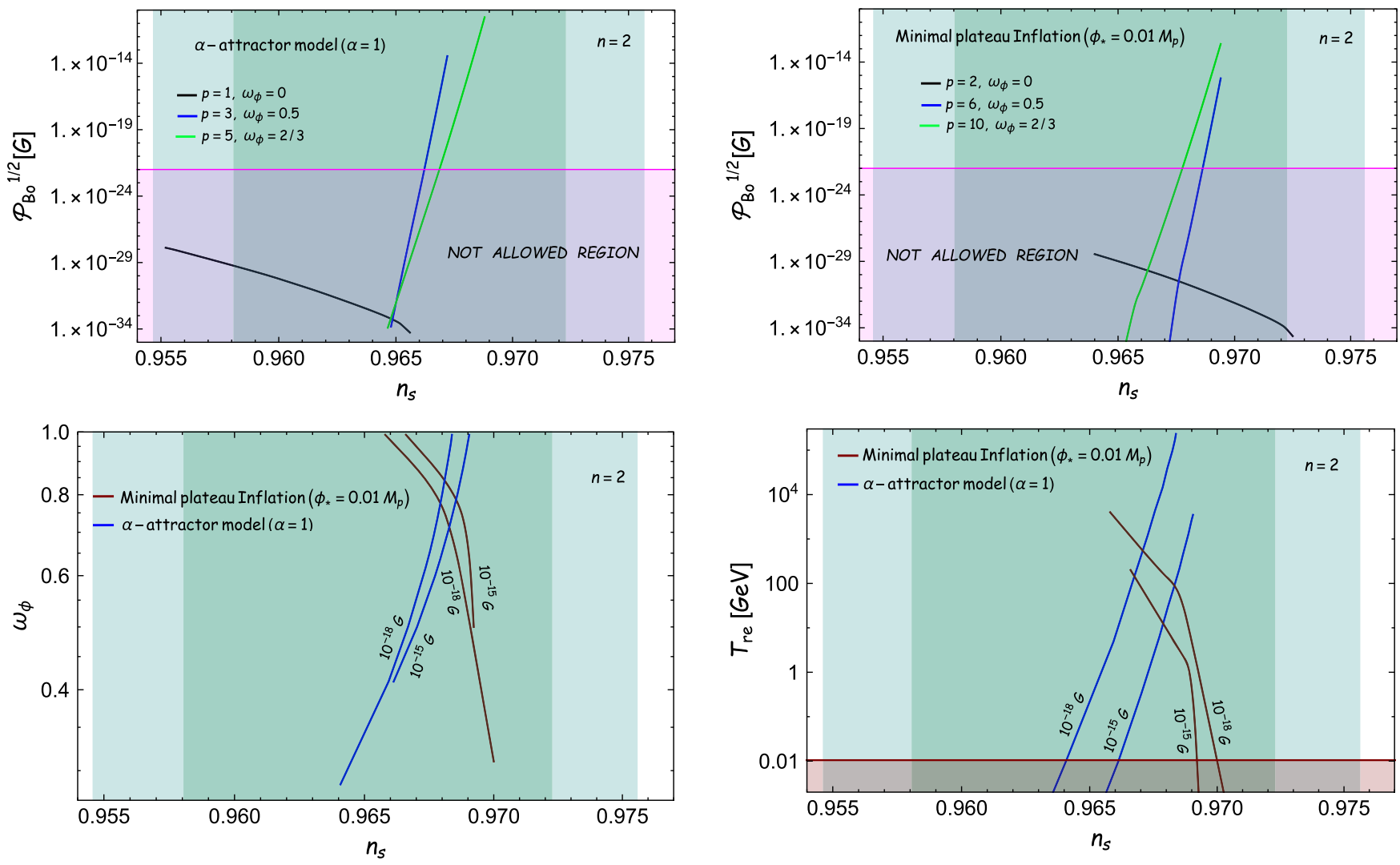

FIG. 3. In the first two plots, we have shown the variation of the present-day magnetic field as a function of the spectral index within the minimum and maximum values of the reheating temperature for different inflationary models, considering the perturbative dynamics of reheating with inflaton equation of state $\left[\omega_{\phi}=\left(0, \frac{1}{2}, \frac{2}{3}\right)\right]$. The light pink region indicates the not allowed region as the strength of the magnetic field in that region is less than $10^{-22} \mathrm{G}$ (observed value of the magnetic field on scales $\approx 1 \mathrm{Mpc}$ must be $B_{\text {cosmic }}^{0}>10^{-22} \mathrm{G}$ [21]). We plot on the lower left side the variation of the inflaton equation of state $\omega_{\phi}$ as a function of the spectral index for two different values of the magnetic field $\mathcal{P}_{B_{0}}^{1 / 2}=\left(10^{-15}, 10^{-18}\right) \mathrm{G}$. On the lower right side, we have plotted the variation of reheating temperature as a function of the spectral index with the same fixed values of the $\mathcal{P}_{B_{0}}^{1 / 2}$. The light brown region indicates the reheating temperature $\left(T_{r e}\right)$ below $10^{-2} \mathrm{GeV}$, which would ruin the predictions of big bang nucleosynthesis (BBN). 
TABLE VIII. Different inflationary models and their associated bounds on reheating temperature $\left(T_{r e}\right)$ and present magnetic field $\left(\mathcal{P}_{B 0}^{\frac{1}{2}}\right)$, measured in units of Gauss (Perturbative reheating dynamics).

\begin{tabular}{lccccc}
\hline \hline & \multicolumn{2}{c}{$\alpha$-attractor model $(\alpha=1)$} & & \multicolumn{2}{c}{ Minimal plateau model $\left(\phi_{*}=0.01 M_{p}\right)$} \\
\cline { 2 - 3 } \cline { 5 - 5 } Parameters & $\omega_{\phi}=0.5$ & $\omega_{\phi}=2 / 3$ & & $\omega_{\phi}=0.5$ & $\omega_{\phi}=2 / 3$ \\
\hline $\mathcal{P}_{\beta}^{\frac{1}{2}}($ minimum $)$ & $10^{-22}$ & $10^{-22}$ & & $10^{-22}$ & $10^{-22}$ \\
$\mathcal{P}_{B 0}^{2}($ maximum $)$ & $3.4 \times 10^{-14}$ & $2.9 \times 10^{-11}$ & & $6.0 \times 10^{-16}$ & $2.3 \times 10^{-13}$ \\
$n_{s}^{\min }$ & 0.9662 & 0.9669 & & 0.9687 & 0.9678 \\
$n_{s}^{\max }$ & 0.9672 & 0.9688 & & 0.9694 & 0.9694 \\
$T_{r e}^{\min }(\mathrm{GeV})$ & $10^{-2}$ & $10^{-2}$ & & $10^{-2}$ & $10^{-2}$ \\
$T_{r e}^{\max }(\mathrm{GeV})$ & $2.4 \times 10^{4}$ & $2.1 \times 10^{6}$ & & $1.7 \times 10^{3}$ & $2.3 \times 10^{4}$ \\
\hline \hline
\end{tabular}

inflaton equation of state. For example, as long as the scaleinvariant power spectra model is concerned, $\omega_{\phi}=0$ cannot produce the required strength of the magnetic field, which is indicating the fact that inflaton field with quadratic potential near its minimum is not compatible with the observation. However, assuming a higher inflaton equation of state $\omega_{\phi}=(p-2) /(p+2)>\frac{1}{3}$ the observable magnetic field strength can be successfully generated within the perturbative reheating framework shown in the last two plots of Fig. 3. Further, considering the magnetic field strength within the observational limit, the maximum and minimum allowed values of the spectral index $n_{s}$, the inflaton equation of state $\omega_{\phi}$ and the associated reheating temperature are provided in Tables V, VIII for different inflationary models.

With this general discussion, in the following sections we consider various inflationary models and discuss about their quantitative predictions in detail.

\section{B. Natural inflation [79]}

The form of the natural inflation potential is given as,

$$
V(\phi)=\Lambda^{4}\left[1-\cos \left(\frac{\phi}{f}\right)\right],
$$

here $\Lambda$ is the height of the potential, and $f$ is the width, also known as the axion decay width. To fit this model with Planck data, this model needs a super-Planckian value of axion decay constant. This is why we have taken $f=$ $50 M_{p}$ for our numerical analysis purpose. The overall scale of the inflation set by $\Lambda$ is fixed by the CMB normalization.

To connect the primordial magnetic field with those observed in the present universe through reheating dynamics, we need to define inflationary $e$-folding number $N_{k}$ and tensor-to -scaler ratio $r_{k}$. In the framework of the natural inflation model, the inflationary parameters $N_{k}$ and $r_{k}$ in terms of $n_{s}$ are defined as

$$
\begin{aligned}
N_{k} & =\frac{f^{2}}{M_{p}^{2}} \ln \left(\frac{2 f^{2}\left(f^{2}\left(1-n_{s}\right)+M_{p}^{2}\right)}{\left(2 f^{2}+M_{p}^{2}\right)\left(f^{2}\left(1-n_{s}\right)-M_{p}^{2}\right)}\right), \\
r_{k} & =4\left(\frac{f^{2}\left(1-n_{s}\right)-M_{p}^{2}}{f^{2}}\right) .
\end{aligned}
$$

For the perturbative reheating dynamics, the initial conditions to solve the Boltzmann equations are set to be

$$
\begin{aligned}
& \Phi(A=1)=\frac{3}{2} \frac{2 \Lambda^{4} M_{p}^{2}}{\left(2 f^{2}+M_{p}^{2}\right) m_{\phi}^{4}}, \\
& R(A=1)=\frac{3\left(\omega_{\mathrm{eff}}-\omega_{\phi}\right)}{1-3 \omega_{\mathrm{eff}}} \Phi(A=1),
\end{aligned}
$$

where

$$
\Lambda=\left(\frac{3 \pi^{2} M_{p}^{2} A_{s}\left(f^{4}\left(1-n_{s}\right)^{2}-M_{p}^{4}\right)}{2 f^{2}}\right)^{\frac{1}{4}}, \quad m_{\phi}=\frac{\Lambda^{2}}{f} .
$$

Here, the primordial scalar amplitude of the inflationary scalar fluctuation is $A_{\delta \phi} \sim 2.19 \times 10^{-9}$ from Planck [1]. As we have the connection relation [Eqs. (58), (68)] between the reheating parameters and the parameters of the magnetogenesis model, in the following, we discuss their implications and various constraints for inflationary magnetogenesis model with $n=2$ for two aforementioned reheating scenarios.

In the first panel of the Fig. 2, for PLANCK central value of $n_{s}=0.9649$, the reheating equation of state $\omega_{\text {eff }}$ has to be bounded within a very small window $(0.294,0.304)$ which is close to the radiation equation of state. The upper bound is associated with the BBN limit of $T_{r e} \sim 10^{-2} \mathrm{GeV}$. From the third panel of Fig. 2, one observes that with the decreasing magnetic field, the maximum allowed reheating temperature is increasing, which seems intuitively obvious as reheating $e$-folding is accordingly decreasing. All the above results are for the case I reheating scenario.

For the perturbative reheating (case II) model with $\omega_{\phi}=0$, the large-scale magnetic field's maximum strength is $\sim 10^{-28} \mathrm{G}$, which is much smaller compared to the observational limit. Hence we can conclude that the natural inflation model is not compatible with the large-scale magnetic field observation within the perturbative reheating framework. This is attributed to the fact that axion potential is quadratic in nature near its minimum.. 


\section{C. $\alpha$-attractor model [80]}

$\alpha$-attractor is class of inflation model that unifies a large number of the existing inflationary models proposed in [80]. Inflaton potential in its canonical form is parametrized as,

$$
V(\phi)=\Lambda^{4}\left[1-e^{\left.-\sqrt{\frac{2}{3 a M_{p}}}\right]^{2 p} .}\right.
$$

The canonical property of this class of models is their universal prediction of inflationary observables $\left(n_{s}, r\right)$ which are Planck observation compatible [1,81] for a large range of $(p, \alpha)$ values. Here the mass scale $\Lambda$ is fixed from $\mathrm{CMB}$ normalization. Interestingly HiggsStarobinsky model is one member of the above class with $[82,83] p=1, \alpha=1$. We will focus on this particular model for our following discussion. For this model, the relation among the inflationary $e$-folding number, $N_{k}$ and tensor to scalar ratio, $r_{k}$, in terms of $n_{s}$ are expressed as,

$$
\begin{aligned}
N_{k} & =\frac{3 \alpha}{4 p}\left[e^{\sqrt{\frac{2}{3 \alpha M_{p}}}}-e^{\sqrt{\frac{2}{3 \alpha} \Phi_{\text {end }}}}-\sqrt{\frac{2}{3 \alpha}} \frac{\left(\Phi_{k}-\Phi_{\mathrm{end}}\right)}{M_{p}}\right], \\
r_{k} & =\frac{64 p^{2}}{3 \alpha\left(e^{\sqrt{\frac{2}{3 \alpha M_{p}}}}-1\right)^{2}} .
\end{aligned}
$$

In addition to that, the initial conditions to solve the Boltzmann equations for different energy components, considering the perturbative reheating model in the context of the present scenario, can be expressed as

$\Phi(A=1)=\frac{3}{2} \Lambda^{4}\left(\frac{2 p}{2 p+\sqrt{3 \alpha}}\right)^{2 p}, \quad R(A=1)=0$,

where

$$
\begin{aligned}
\Lambda= & M_{p}\left(\frac{3 \pi^{2} r A_{s}}{2}\right) \\
& \times\left[\frac{2 p(1+2 p)+\sqrt{4 p^{2}+6 \alpha(1+p)\left(1-n_{s}\right)}}{4 p(1+p)}\right]^{\frac{p}{2}} .
\end{aligned}
$$

We consider $\alpha$-attractor model with $\alpha=1$ for two different reheating scenarios described before. Detail prediction and model constraints can be read from Figs. 2, 3 and Tables III, IV, VIII. Taking a particular central value of the scalar spectral, $n_{s}=0.9649$, the effective equation of state is constrained within a very narrow range $(0.302,0.310)$. As has been mentioned already and also seen for the axion model, large scale magnetic field appeared to be consistent with low reheating temperature with maximum value $\sim 1 \mathrm{TeV}$ for $\mathcal{P}_{B_{0}}^{1 / 2} \sim 10^{-22} \mathrm{G}$. The above conclusion are based on instantaneous reheating scenario.

For perturbative case, $\omega_{\phi}=0$ turned out to be observationally unsuitable. However, for the model with $p=(3,5)$ where the inflaton equation states assume $\omega_{\phi}=\left(0.5, \frac{2}{3}\right)$ accordingly, are observed to be potentially consistent with observation. Most importantly the perturbative reheating supports higher value of reheating temperature $T_{r e}^{\max } \sim\left(2.4 \times 10^{4}, 2.1 \times 10^{6}\right) \mathrm{GeV}$ for magnetic field strength $\mathcal{P}_{B_{0}}^{\frac{1}{2}} \sim 10^{-22} \mathrm{G}$, with $\omega_{\phi}=\left(0.5, \frac{2}{3}\right)$ respectively. Quantitative values of all the reheating parameters and inflationary parameters $\left(n_{s}, \omega_{\phi}, T_{r e}\right)$ corresponding to different sample values of $\mathcal{P}_{B_{0}}^{1 / 2}$ are provided in Table V. This can provide us clear picture of the viability of the $\alpha$ attractor model in the context of inflationary magnetogenesis scenarios.

\section{Minimal plateau model}

In this section, we will introduce a special class of the inflationary model, the minimal plateau models proposed in [84]. The potential of this type of model is a nonpolynomial modification to the simple power-law potential $\phi^{n}$ and is given by

$$
V_{\min }=\Lambda \frac{m^{4-p} \phi^{p}}{1+\left(\frac{\phi}{\phi_{*}}\right)^{p}}
$$

Where $m$ and $\phi_{*}$ are two mass scales. The parameter $\lambda$ and the scale $m$ are fixed from WMAP normalization [85]. Only even values of the index $p$ are taken, as was the case for the chaotic inflation model. The new scale $\phi_{*}$ can be shown to fix the scalar spectral index, and the scalar-to tensor ratio within the observational limit from Planck $[1,81]$. For the numerical purpose, we choose $\phi_{*}=0.01 M_{p}$. Before we go to the quantitative discussion to acquire one to one correspondence between the reheating parameters $\left(\omega_{\text {eff }}\left(\omega_{\phi}\right), T_{r e}\right)$ with $\mathcal{P}_{B_{0}}^{1 / 2}$, let us point out the usual inflationary parameters for this class of minimal models we discussed. The inflationary parameters $N_{k}$ and $r_{k}$ can be written as,

$$
r_{k}=\frac{8 M_{p}^{2} p^{2}}{\phi^{2}\left(1+\left(\frac{\phi}{\phi_{*}}\right)^{p}\right)^{2}}, \quad N_{k}=\int_{\phi_{k}}^{\phi_{\mathrm{end}}}-\frac{\phi\left(\phi_{*}^{p}+\phi^{p}\right)}{p M_{p}^{2} \phi_{*}^{p}} d \phi
$$

Moreover, similar to the other inflation model, the initial conditions to solve the differential equation in the case of the perturbative reheating model are set as

$$
\Phi(A=1)=\frac{3}{2} \frac{V_{\text {end }}}{m_{\phi}^{4}}, \quad R(A=1)=0,
$$

where 


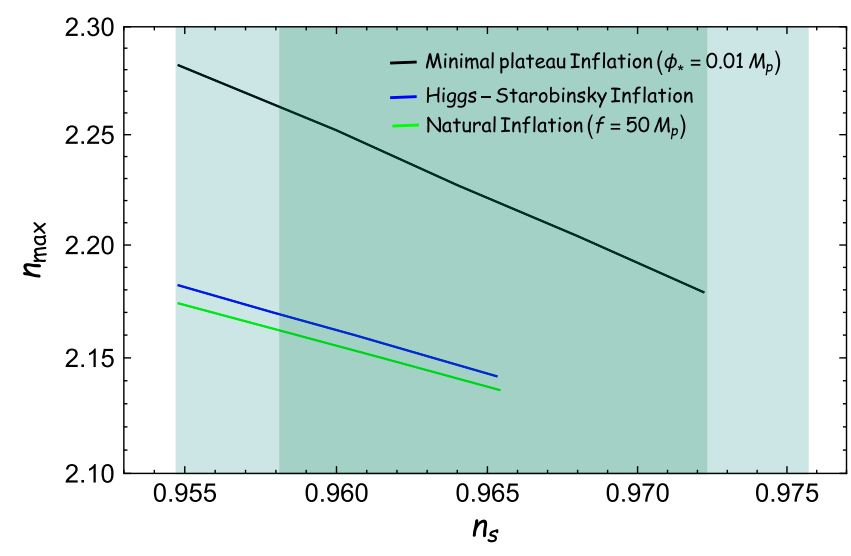

FIG. 4. We plot the variation of maximum value of $n\left(n_{\max }\right)$ as a function of spectral index within $2 \sigma$ range, $n_{s}$ from Planck [1] for different inflationary model. In this plot the maximum values of the spectral index correspond to the instantaneous reheating condition $N_{r e} \rightarrow 0$.

$$
\begin{aligned}
V_{\mathrm{end}} & =\frac{m^{4-p} \phi_{\mathrm{end}}^{p}}{1+\left(\frac{\phi_{\mathrm{end}}}{\phi_{*}}\right)^{p}} \\
m & =\left(\frac{3 \pi^{2} M_{p}^{4} r_{k} A_{s}}{2 \Lambda \phi_{k}^{p}}\left(1+\left(\frac{\phi_{k}}{\phi_{*}}\right)^{p}\right)\right)^{\frac{1}{4-p}} .
\end{aligned}
$$

We set $\Lambda=1$.

Details constraints on the reheating parameter space can be read from Figs. 2, 3 and Tables VI, VII, VIII.

Let us point out that for the minimal-inflation model, the constraints on the reheating parameters in terms of scalar spectral index and large scale magnetic field qualitatively differ from that of the $\alpha$-attractor model shown in Fig. 3. This difference can be attributed to the qualitatively different nature of the potential near its minima and the initial condition at the beginning of reheating. The numerical values and their constraints of reheating parameters $\left(\omega_{\text {eff }}, T_{\text {re }}\right)$ for different sample values of $\mathcal{P}_{B_{0}}^{1 / 2}$ cane be read from the Table VII for instantaneous reheating case, and Table VIII, third panel of Fig. 3 for perturbative reheating case.

\section{E. Constraining magnetogensis model: Maximum possible value of $\boldsymbol{n}$ and corresponding reheating temperature $T_{r e}$}

The scaling properties of the large-scale magnetic field as a function of momentum are not particularly well understood from the observation point of view. Throughout the paper, though, we have emphasized two special cases of interest related to scale-invariant electric field $(n=2)$ and scale-invariant magnetic field $(n=3)$. Any other value of $n$, which is not special, could have observational relevance in the near future. Keeping this motivation in mind, in this section, we study the possible range of $n$ values, which can give rise to the observable strength of the magnetic field. As has already been described before, the direct constraint on the value of $n$ will come from the strong coupling and the backreaction problem leading to the fact that $n$ should lie within $0 \leq n \leq n_{\max }$, where $n_{\max }$ can be determined from Eqs. (44), (45). Our primary goal of this section is to pinpoint the value of $n_{\max }$ for different models under consideration. From the Fig. 4, we can clearly see that the permissible range of $n_{\max }$ for various inflationary models should lie approximately within (2.15-2.3). As has been observed before, conventional magnetogenesis scenarios are generically viable for low-scale inflationary models or, in other words, low reheating temperature regimes. This turned to be untrue for higher $n \sim n_{\max }$. In order to calculate reheating temperature associated with the maximum possible value of the $n$, we consider two sample value of the spectral index $n_{s}=\left(n_{s}^{\text {central }}=0.9649, n_{s}^{\max }\right)$. Irrespective of the inflationary models under consideration, reheating temperature can be observed as high as $\sim 10^{7} \mathrm{GeV}$ without any backreaction problem. In Table IX, we have provided the limiting values of the $\left(\omega_{\text {eff }}, T_{r e}, \mathcal{P}_{B_{0}}^{1 / 2}\right)$ for two different values of the scalar spectral index $n_{s}$. To this end, let us point out an important result of our analysis that the minimal plateau model can be observationally discarded as it predicts reheating temperature less than BBN limit $\left(10^{-2} \mathrm{Gev}\right)$ for $0 \leq \omega_{\text {eff }} \leq \frac{1}{3}$.

TABLE IX. Different inflationary models and their associated bounds on reheating temperature $\left(T_{r e}\right)$ and present magnetic field $\left(\mathcal{P}_{B 0}^{\frac{1}{2}}\right)$

\begin{tabular}{|c|c|c|c|c|c|}
\hline \multirow[b]{2}{*}{ Parameters } & \multicolumn{2}{|c|}{ Axion $\left(f=50 M_{p}\right)$} & \multicolumn{2}{|c|}{ Higgs-Starobinsky } & \multirow{2}{*}{$\frac{\text { Minimal plateau }\left(\phi_{*}=0.01 M_{p}\right)}{n_{s}^{\max }(0.9722)}$} \\
\hline & $n_{s}^{\max }(0.9654)$ & $n_{s}^{\text {central }}$ & $n_{s}^{\max }(0.9653)$ & $n_{s}^{\text {central }}$ & \\
\hline$n_{\max }$ & 2.136 & 2.138 & 2.142 & 2.144 & 2.179 \\
\hline $\mathcal{P}_{B 0}^{\frac{1}{2}}($ minimum $)$ & $10^{-22}$ & $10^{-22}$ & $10^{-22}$ & $10^{-22}$ & $10^{-22}$ \\
\hline $\mathcal{P}_{B 0}^{\frac{1}{2}}($ maximum $)$ & $2.6 \times 10^{-13}$ & $5.5 \times 10^{-14}$ & $4.2 \times 10^{-14}$ & $1.3 \times 10^{-14}$ & $2.3 \times 10^{-14}$ \\
\hline$T_{r e}^{\min }(\mathrm{GeV})$ & $10^{-2}$ & $10^{-2}$ & $10^{-2}$ & $10^{-2}$ & $10^{-2}$ \\
\hline$T_{r e}^{\max }(\mathrm{GeV})$ & $2.3 \times 10^{7}$ & $5.8 \times 10^{6}$ & $4.9 \times 10^{6}$ & $1.4 \times 10^{6}$ & $2 \times 10^{6}$ \\
\hline$\omega_{\mathrm{eff}}^{\min }$ & 0.3281 & 0.2762 & 0.3297 & 0.2904 & 0.3218 \\
\hline$\omega_{\mathrm{eff}}^{\max }$ & 0.3309 & 0.3042 & 0.3315 & 0.3120 & 0.3274 \\
\hline
\end{tabular}
(measured in units of Gauss), considering maximum allowed value of the coupling parameter $n_{\max }$. 


\section{SUMMARY AND DISCUSSION}

Among all the well-known magnetogenesis scenarios, inflationary magnetogenesis is a well-motivated and simple mechanism to explain the large-scale magnetic field of our universe. In the present paper, we specifically focus on the effect of reheating phase on the evolution of the inflationary magnetic field. In this regard, Faraday's effect of magnetic induction plays an important role. The primary assumption behind this mechanism to act during reheating is the negligible electrical conductivity. As a result, the strong electric field can survive during this phase and is dynamically converted into the magnetic field. Hence the resulting primordial magnetic field energy density does not evolve as radiation energy density $\left(1 / a^{4}\right)$ during this phase; instead, it evolves as $\propto 1 / H^{2} a^{6}$ till the end of the reheating phase. After the end of the reheating electric field dies out fast due to the large electric conductivity and comoving magnetic energy density freezes out until today. Considering this physical effect, the present-day amplitude of the magnetic field originated during inflation becomes observationally viable with a specific set of reheating parameters. Our motivation for this work is to figure out these constraints on the reheating parameters, which directly shape the inflation and magnetogenesis models.

We have derived explicit connection among the inflationary $\left(n_{s}\right)$, reheating $\left(\omega_{\text {eff }}, T_{r e}\right)$, and magnetogenesis $(n)$ model parameters assuming two different reheating scenarios. The large-scale magnetic field gives rise to stringent constraints on both the reheating parameters depending upon the models under consideration. Considering both backreaction and strong coupling problems into account, the maximum allowed value of $n$ would be $n_{\max } \sim 2.3$ for all the models such as natural inflation and HiggsStarobinsky, and minimal plateau model. The associated maximum allowed value of $n$, the reheating temperature has been shown to lie within a wide range of $\left(10^{-2}, 10^{7}\right) \mathrm{GeV}$.

We also have some definite predictions once we fix the model and the large-scale magnetic field. For reheating scenario with a constant equation of state $\omega_{\text {eff }}$, once we fixed the scalar spectral index, a unique value of $\omega_{\text {eff }}$ is predicted associated with the specific choice of the present-day magnetic field. This unique $\omega_{\text {eff }}$, further predicts a specific value of reheating temperature $T_{r e}$. The quantitative values of those parameters for $n=2$ magnetogenesis model are provided in the Tables I, III, and VI. As reheating and inflationary parameters are connected, the present-day strength of the magnetic field also provides possible limits on the inflationary scalar spectral index. This extra bound from inflationary magnetogenesis essentially narrows down the possible value of $n_{s}$ within the $1 \sigma$ range of $n_{s}=0.9649 \pm 0.0042$ (68\% CL, Planck TT, TE, EE + lowE + lensing) from Planck [1] (shown in Tables II, IV, and VII for different inflationary models). Model-independent constraint on the effective equation of state is obtained as $\left(0.15<\omega_{\text {eff }}<\frac{1}{3}\right)$. One of the main drawbacks of describing the reheating dynamics by a constant $\omega_{\text {eff }}$ is that it does not enable us to decode the nature of the inflation potential near its minimum. This drawback motivated us to consider the reheating in the perturbative framework where inflation equation of state is described by $\omega_{\phi} \equiv$ $(p-2) /(p+2)$ with inflaton potential $V(\phi) \sim \phi^{p}$ near its minimum.

For the perturbative reheating scenario, the inflation equation of state $\omega_{\phi} \gtrsim 0.28$ is found to be observationally viable for $\mathcal{P}_{B 0}^{\frac{1}{2}} \gtrsim 10^{-18} \mathrm{G}$. Increasing the lower limit of the observable magnetic field will further increase the lower limit of $\omega_{\phi}$. We think it is extremely interesting in the context of inflation model building. This observation provides a strong constraint on the possible form of the inflaton potential near its minimum with $p \gtrsim 3.6$ considering the aforesaid observable limit of the present-day magnetic field strength. Furthermore, if one considers the higher equation of state, the high scale inflation model with reheating temperature as high as $10^{7} \mathrm{GeV}$ is possible. The main outcomes of the perturbative analysis for the different inflationary models are shown in Tables V, VIII. In this paper, we develop the methodology to probe the reheating phase, combining both the present value of the large-scale magnetic field and CMB anisotropy. For that, we considered the simplest magnetogenesis scenario, the so-called Ratra's model. Different magnetogenesis models can be studied straightforwardly following the formalism developed in this paper to probe reheating phase. This has already been applied in the recent papers [86,87]. The only differences that may arise are in the allowed reheating and inflationary parameters space. However, to distinguish those models, we need more physical observable such as primordial gravitational wave [88], dark matter, etc. Our eventual plan in the future is to obtain some universal constraints on the reheating and inflationary parameters taking into account various cosmological observables just mentioned.

\section{ACKNOWLEDGMENTS}

We would like to thank the HEP and Gravity groups at IIT Guwahati for useful discussions. We thank the anonymous referees for useful comments which help us to improve our work. M. R. H. and S. P. acknowledge financial assistance from MHRD, Government of India. 
[1] Y. Akrami et al. (Planck Collaboration), Astron. Astrophys. 641, A10 (2020).

[2] É. Aubourg, S. Bailey, J. E. Bautista, F. Beutler, V. Bhardwaj, D. Bizyaev, M. Blanton, M. Blomqvist, A. S. Bolton, and J. Bovy et al., Phys. Rev. D 92, 123516 (2015).

[3] D. Baumann and H. V. Peiris, Adv. Sci. Lett. 2, 105 (2009).

[4] J. A. Vázquez, L. E. Padilla, and T. Matos, arXiv:1810 .09934.

[5] M. Kawasaki, K. Kohri, and N. Sugiyama, Phys. Rev. D 62 , 023506 (2000).

[6] G. Steigman, Annu. Rev. Nucl. Part. Sci. 57, 463 (2007).

[7] B. D. Fields, P. Molaro, and S. Sarkar, Chin. Phys. C 38, 339 (2014).

[8] M. Kawasaki, K. Kohri, and N. Sugiyama, Phys. Rev. Lett. 82, 4168 (1999).

[9] D. Baumann, arXiv:0907.5424.

[10] A. Riotto, ICTP Lect. Notes Ser. 14, 317 (2003), arXiv: hep-ph/0210162.

[11] M. R. Haque, D. Maity, and P. Saha, Phys. Rev. D 102, 083534 (2020).

[12] L. Dai, M. Kamionkowski, and J. Wang, Phys. Rev. Lett. 113, 041302 (2014).

[13] J. Martin and C. Ringeval, Phys. Rev. D 82, 023511 (2010).

[14] D. Maity and P. Saha, Phys. Rev. D 98, 103525 (2018).

[15] D. Maity and P. Saha, J. Cosmol. Astropart. Phys. 07 (2019) 018.

[16] C. Caprini and D. G. Figueroa, Classical Quantum Gravity 35, 163001 (2018).

[17] K. Nakayama, S. Saito, Y. Suwa, and J. Yokoyama, Classical Quantum Gravity 0806, 020 (2008).

[18] T. Kobayashi and M. S. Sloth, Phys. Rev. D 100, 023524 (2019).

[19] R. J. Ferreira, R. K. Jain, and M. S. Sloth, J. Cosmol. Astropart. Phys. 10 (2013) 004.

[20] B. Ratra, Astrophys. J. Lett. 391, L1 (1992).

[21] J. Martin and J. Yokoyama, J. Cosmol. Astropart. Phys. 01 (2008) 025.

[22] K. Subramanian, arXiv:astro-ph/9609123.

[23] T. Kobayashi and N. Afshordi, J. High Energy Phys. 10 (2014) 166.

[24] T. Fujita and R. Namba, Phys. Rev. D 94, 043523 (2016).

[25] Y. Shtanov, J. Cosmol. Astropart. Phys. 10 (2019) 008.

[26] P. Qian and Z. K. Guo, Phys. Rev. D 93, 043541 (2016).

[27] M. S. Turner and L. M. Widrow, Phys. Rev. D 37, 2743 (1988).

[28] K. Bamba, Phys. Rev. D 91, 043509 (2015).

[29] V. Demozzi, V. Mukhanov, and H. Rubinstein, J. Cosmol. Astropart. Phys. 08 (2009) 025.

[30] R. Sharma, S. Jagannathan, T. R. Seshadri, and K. Subramanian, Phys. Rev. D 96, 083511 (2017).

[31] D. Grasso and H. R. Rubinstein, Phys. Rep. 348, 163 (2001).

[32] P. P. Kronberg, Rep. Prog. Phys. 57, 325 (1994).

[33] L. M. Widrow, Rev. Mod. Phys. 74, 775 (2002).

[34] Z. R. Wang, S. Q. Xi, R. Y. Liu, R. Xue, and X. Y. Wang, Phys. Rev. D 101, 083004 (2020).

[35] K. Takahashi, M. Mori, K. Ichiki, and S. Inoue, Astrophys. J. Lett. 744, L7 (2012).

[36] K. Takahashi, M. Mori, K. Ichiki, S. Inoue, and H. Takami, Astrophys. J. Lett. 771, L42 (2013).
[37] J. Asplund, G. Jóhannesson, and A. Brandenburg, Astrophys. J. 898, 124 (2020).

[38] M. Kachelriess and B. C. Martinez, Phys. Rev. D 102, 083001 (2020).

[39] A. Neronov and I. Vovk, Science 328, 73 (2010).

[40] W. Essey, S. Ando, and A. Kusenko, Astropart. Phys. 35, 135 (2011).

[41] J. D. Finke, L. C. Reyes, M. Georganopoulos, K. Reynolds, M. Ajello, S. J. Fegan, and K. McCann, Astrophys. J. 814, 20 (2015).

[42] S. Saga, H. Tashiro, and S. Yokoyama, Phys. Rev. D 98, 083518 (2018).

[43] K. Takahashi, M. Mori, K. Ichiki, S. Inoue, and H. Takami, Astrophys. J. Lett. 771, L42 (2013).

[44] T. A. Dzhatdoev, E. I. Podlesnyi, and I. A. Vaiman, Phys. Rev. D 102, 123017 (2020).

[45] K. Jedamzik and A. Saveliev, Phys. Rev. Lett. 123, 021301 (2019).

[46] A. Boyarsky, J. Frohlich, and O. Ruchayskiy, Phys. Rev. Lett. 108, 031301 (2012).

[47] J. H. Matthews, A. R. Bell, K. M. Blundell, and A. T. Araudo, Mon. Not. R. Astron. Soc. 469, 1849 (2017).

[48] A.Z. Dolginov and M. E. Katz, Phys. Rep. 239, 285 (1994).

[49] H. Sol, G. Pelletier, and E. Asseo, Mon. Not. R. Astron. Soc. 237, 411 (1989).

[50] A. Brandenburg, K. Enqvist, and P. Olesen, Phys. Rev. D 54, 1291 (1996).

[51] S. Sur, A. Shukurov, and K. Subramanian, Mon. Not. R. Astron. Soc. 377, 874 (2007).

[52] D. Boyanovsky, H. J. de Vega, and M. Simionato, Phys. Rev. D 67, 123505 (2003).

[53] J. M. Wagstaff and R. Banerjee, J. Cosmol. Astropart. Phys. 01 (2016) 002.

[54] T. Vachaspati, Phys. Lett. B 265, 258 (1991).

[55] Y. Zhang, T. Vachaspati, and F. Ferrer, Phys. Rev. D 100, 083006 (2019).

[56] J. L. Cook, E. Dimastrogiovanni, D. A. Easson, and L. M. Krauss, J. Cosmol. Astropart. Phys. 04 (2015) 047.

[57] T. Patel, H. Tashiro, and Y. Urakawa, J. Cosmol. Astropart. Phys. 01 (2020) 043.

[58] V. Domcke and K. Mukaida, J. Cosmol. Astropart. Phys. 11 (2018) 020.

[59] P. Adshead, J. T. Giblin, T. R. Scully, and E. I. Sfakianakis, J. Cosmol. Astropart. Phys. 10 (2016) 039.

[60] K. Atmjeet, I. Pahwa, T. R. Seshadri, and K. Subramanian, Phys. Rev. D 89, 063002 (2014).

[61] J. D. Barrow, C. G. Tsagas, and K. Yamamoto, Phys. Rev. D 86, 023533 (2012).

[62] A. Neronov, A. M. Taylor, C. Tchernin, and I. Vovk, Astron. Astrophys. 554, A31 (2013).

[63] T. Vachaspati, Phys. Rev. D 95, 063505 (2017).

[64] T. Fujita and S. Mukohyama, J. Cosmol. Astropart. Phys. 10 (2012) 034.

[65] R. J. Ferreira, R. K. Jain, and M. S. Sloth, J. Cosmol. Astropart. Phys. 06 (2014) 053.

[66] D. Green and T. Kobayashi, J. Cosmol. Astropart. Phys. 03 (2016) 010.

[67] R. Z. Ferreira, J. Ganc, J. Norea, and M. S. Sloth, J. Cosmol. Astropart. Phys. 04 (2016) 039. 
[68] M. Gasperini, M. Giovannini, and G. Veneziano, Phys. Rev. Lett. 75, 3796 (1995).

[69] K. Bamba and J. Yokoyama, Phys. Rev. D 69, 043507 (2004).

[70] S. Kanno, J. Soda, and M. A. Watanabe, J. Cosmol. Astropart. Phys. 12 (2009) 009.

[71] N. Barnaby, R. Namba, and M. Peloso, Phys. Rev. D 85, 123523 (2012).

[72] T. Suyama and J. Yokoyama, Phys. Rev. D 86, 023512 (2012).

[73] R. K. Jain and M. S. Sloth, Phys. Rev. D 86, 123528 (2012).

[74] R. K. Jain and M. S. Sloth, J. Cosmol. Astropart. Phys. 02 (2013) 003.

[75] S. Nurmi and M. S. Sloth, J. Cosmol. Astropart. Phys. 07 (2014) 012.

[76] T. Fujita and S. Yokoyama, J. Cosmol. Astropart. Phys. 03 (2014) 013.

[77] J. Ganc and M. S. Sloth, J. Cosmol. Astropart. Phys. 08 (2014) 018.
[78] V. Mukhanov, Physical Foundations of Cosmology (Cambridge University Press, Cambridge, England, 2005).

[79] K. Freese, J. A. Frieman, and A. V. Olinto, Phys. Rev. Lett. 65, 3233 (1990).

[80] R. Kallosh and A. Linde, J. Cosmol. Astropart. Phys. 07 (2013) 002; R. Kallosh, A. Linde, and D. Roest, J. High Energy Phys. 11 (2013) 198.

[81] P. A. R. Ade et al. (Planck Collaboration), Astron. Astrophys. 594, A20 (2016).

[82] F. L. Bezrukov and M. Shaposhnikov, Phys. Lett. B 659, 703 (2008).

[83] A. A. Starobinsky, Phys. Lett. 91B, 99 (1980).

[84] D. Maity and P. Saha, Classical Quantum Gravity 36, 045010 (2019).

[85] E. Komatsu et al. (WMAP Collaboration), Astrophys. J. Suppl. 192, 18 (2011).

[86] D. Maity, S. Pal, and T. Paul, arXiv:2103.02411.

[87] K. Bamba, E. Elizalde, S. D. Odintsov, and T. Paul, J. Cosmol. Astropart. Phys. 04 (2021) 009.

[88] R. Sharma, arXiv:2102.09358. 